\title{
Knowing the unknowns : uncertainties in simple estimators of galactic dynamical masses
}

\section{Campbell, David J. R.}

2017-08

Campbell , D J R , Frenk, C S , Jenkins , A, Eke , V R, Navarro , J F , Sawala , T , Schaller , M , Fattahi , A , Oman , K A \& Theuns , T 2017 , ' Knowing the unknowns : uncertainties in simple estimators of galactic dynamical masses ' , Monthly Notices of the Royal Astronomical Society , vol. 469 , no. 2 , pp. 2335-2360 . https://doi.org/10.1093/mnras/stx975

http://hdl.handle.net/10138/215225

https://doi.org/10.1093/mnras/stx975

unspecified

publishedVersion

Downloaded from Helda, University of Helsinki institutional repository.

This is an electronic reprint of the original article.

This reprint may differ from the original in pagination and typographic detail.

Please cite the original version. 


\title{
Knowing the unknowns: uncertainties in simple estimators of galactic dynamical masses
}

\author{
David J. R. Campbell, ${ }^{1 \star}$ Carlos S. Frenk, ${ }^{1}$ Adrian Jenkins, ${ }^{1}$ Vincent R. Eke, ${ }^{1}$ \\ Julio F. Navarro, ${ }^{2} \dagger$ Till Sawala, ${ }^{3}$ Matthieu Schaller, ${ }^{1}$ Azadeh Fattahi, ${ }^{2}$ Kyle A. Oman ${ }^{2}$ \\ and Tom Theuns ${ }^{1}$ \\ ${ }^{1}$ Institute for Computational Cosmology, Department of Physics, Durham University, South Road, Durham DH1 $3 L E$, UK \\ ${ }^{2}$ Department of Physics and Astronomy, University of Victoria, Victoria, BC V8P 5C2, Canada \\ ${ }^{3}$ Department of Physics, University of Helsinki, Gustaf Hällströmin katu 2a, FI-00014 Helsinki, Finland
}

Accepted 2017 April 21. Received 2017 March 21; in original form 2016 March 14

\begin{abstract}
The observed stellar kinematics of dispersion-supported galaxies are often used to measure dynamical masses. Recently, several analytical relationships between the stellar line-of-sight velocity dispersion, the projected (2D) or deprojected (3D) half-light radius and the total mass enclosed within the half-light radius, relying on the spherical Jeans equation, have been proposed. Here, we use the APOSTLE cosmological hydrodynamical simulations of the Local Group to test the validity and accuracy of such mass estimators for both dispersion and rotationsupported galaxies, for field and satellite galaxies, and for galaxies of varying masses, shapes and velocity dispersion anisotropies. We find that the mass estimators of Walker et al. and Wolf et al. are able to recover the masses of dispersion-dominated systems with little systematic bias, but with a $1 \sigma$ scatter of 25 and 23 per cent, respectively. The error on the estimated mass is dominated by the impact of the 3D shape of the stellar mass distribution, which is difficult to constrain observationally. This intrinsic scatter becomes the dominant source of uncertainty in the masses estimated for galaxies like the dwarf spheroidal (dSph) satellites of the Milky Way, where the observational errors in their sizes and velocity dispersions are small. Such scatter may also affect the inner density slopes of dSphs derived from multiple stellar populations, relaxing the significance with which Navarro-Frenk-White profiles may be excluded, depending on the degree to which the relevant properties of the different stellar populations are correlated. Finally, we derive a new optimal mass estimator that removes the residual biases and achieves a statistically significant reduction in the scatter to 20 per cent overall for dispersion-dominated galaxies, allowing more precise and accurate mass estimates.
\end{abstract}

Key words: galaxies: dwarf-galaxies: formation-galaxies: fundamental parametersgalaxies: kinematics and dynamics - dark matter.

\section{INTRODUCTION}

Obtaining reliable dynamical information lies at the heart of many fundamental questions in cosmology and galactic structure. However, in nature we typically only have partial information about the kinematics of observed systems. Dynamical studies are therefore intrinsically underconstrained, and often require the use of various simplifying assumptions. In this study, we are interested in stellar dynamical systems to which the spherical Jeans equation may be applied, such as globular clusters, satellite galaxies and massive

${ }^{\star}$ E-mail: d.j.r.campbell@durham.ac.uk

†Senior CIfAR Fellow. spheroidal galaxies. The use of Jeans analysis to infer the mass distribution of a system whose gravitational potential is traced by stars is complicated by an important degeneracy between the velocity dispersion anisotropy of the stars and the total mass profile of the system. The anisotropy is notoriously difficult to constrain with current data, and therefore studies based on the application of the spherical Jeans equation have led to many ambiguous results. For example, a topic of great interest is whether local satellite galaxies have central cusps or cores in their central dark matter distributions. Studies based on Jeans analysis are often inconclusive, largely due to the anisotropy degeneracy.

An important advance in this subject has been the application of simple estimators to infer the dynamical mass within a sphere of radius equal to the projected (2D) stellar half-light radius, $R_{\mathrm{e}}$ 
(Walker et al. 2009, 2010), or the deprojected (3D) stellar half-light radius, $r_{\text {half }}$ (Wolf et al. 2010). ${ }^{1}$ These estimators make use of only two measured quantities: the stellar line-of-sight velocity dispersion averaged over the whole galaxy, $\left\langle\sigma_{\text {los }}\right\rangle$, and the projected half-light radius. Each estimator can be written in the form,

$M\left(<\lambda R_{\mathrm{e}}\right)=\frac{\mu\left\langle\sigma_{\mathrm{los}}\right\rangle^{2} R_{\mathrm{e}}}{G}$,

where $M(<r)$ is the total mass enclosed within a sphere of radius $r . \lambda$ and $\mu$ are dimensionless parameters, and $G$ is the gravitational constant. These estimators are derived from the spherical Jeans equation, which is valid for spherically symmetric, dispersion-supported, collisionless, stationary systems, in dynamical equilibrium, in the absence of streaming motions. Additional assumptions are applied in the derivation of each estimator, with the assumptions used by Walker et al. (2009) being somewhat more restrictive than those of Wolf et al. (2010). The main finding of these studies is that the total mass within a sphere of radius close to the $2 \mathrm{D}$ or $3 \mathrm{D}$ stellar half-light radius is minimally sensitive to the assumed form of the stellar velocity dispersion anisotropy and the shape of the total mass profile (Wolf et al. 2010 discuss in detail how this relatively tight constraint arises).

Walker \& Peñarrubia (2011) have applied these estimators to apparently distinct stellar subpopulations in the Fornax and Sculptor dwarf spheroidals (dSphs), and have argued for the presence of a central core in the dark matter density profile of these galaxies, with higher confidence for Sculptor than for Fornax. (However, this result has been disputed for Sculptor by Strigari, Frenk \& White 2014.) A further example of the applicability of these mass estimators is the 'too big to fail problem' of the standard $\Lambda$ cold dark matter $(\Lambda \mathrm{CDM})$ cosmological model (Boylan-Kolchin, Bullock \& Kaplinghat 2011), which was predicated on the basis of comparing the circular velocity curves $\left(V_{\mathrm{c}}(r)=\sqrt{G M(<r) / r}\right)$ of dark matter subhaloes drawn from the Aquarius (Springel et al. 2008) and Via Lactea II simulations (Diemand et al. 2008) with the dynamical mass within the 3D half-light radius of the brightest satellites of the Milky Way (MW) according to Wolf et al. (2010).

While the assumptions that underpin the spherical Jeans equation may seem relatively benign, they are not exactly satisfied by all dSphs. For example, it is clear that many galaxies, such as Fornax, are not spherically symmetric. It also seems unlikely that satellites orbiting in the potential well of a host halo are in dynamical equilibrium

The simple mass estimators have been tested on ideal spherically symmetric systems (Walker \& Peñarrubia 2011), simulations of ideal dSphs in a static MW potential (Kowalczyk et al. 2013), stellar distributions placed in subhaloes from dark matter only simulations (Laporte, Walker \& Peñarrubia 2013a), and cosmological zoom simulations of isolated elliptical galaxies (Lyskova et al. 2015). More recently, the first generation of simulations with enough resolution to model the satellite galaxies of the MW reliably, in a realistic cosmological setting, has become available (Grand et al. 2016; Sawala et al. 2016; Wetzel et al. 2016). Such simulations can be used to test the accuracy of equation (1) in estimating the dynamical masses of model galaxies with realistic properties. In this paper, we make use of the APOSTLE simulations of the Local Group (Fattahi et al.

\footnotetext{
${ }^{1} R_{\mathrm{e}}$ is the radius of the circle enclosing half of the total stellar luminosity in projection (effective radius), and $r_{\text {half }}$ is the radius of the sphere enclosing the same luminosity fraction in 3D.
}

2016; Sawala et al. 2016) to study the accuracy of the estimators proposed by Walker et al. (2009) and Wolf et al. (2010).

In Section 2, we describe the mass estimators and the assumptions on which they are based in greater detail. The simulations used in this work are discussed in Section 3. In Section 4, we present general properties of a sample of well-resolved dispersion-dominated galaxies drawn from the simulations, to which we apply the mass estimators in Section 5. The implications of the systematic errors on the estimated masses are discussed in Section 6. In Section 7, we find the set of estimator parameters that optimizes the accuracy of the recovered mass for dispersion-dominated galaxies. Concluding remarks are given in Section 8. In Appendix A, we investigate the accuracy of the estimator of Wolf et al. (2010) when written in terms of the $3 \mathrm{D}$ half-light radius. All simulation results presented in this paper are at redshift zero. The assumed $\Lambda \mathrm{CDM}$ cosmological parameters are given in Section 3.1.

\section{MASS ESTIMATORS}

The spherical Jeans equation relates the circular velocity curve of a spherically symmetric system to the radial distribution and velocity dispersion of a population of tracers that orbit in the gravitational potential (see Section 1 for a list of the assumptions on which this equation is based):

$\frac{G M(<r)}{r}=-\sigma_{r}^{2}(r)\left[\frac{\mathrm{d} \log \rho(r)}{\mathrm{d} \log r}+\frac{\mathrm{d} \log \sigma_{r}^{2}(r)}{\mathrm{d} \log r}+2 \beta(r)\right]$,

where $M(<r)$ is the total mass enclosed within a radius $r$ of the centre, $\rho(r)$ is the density profile of the tracer population, ${ }^{2} \sigma_{r}(r)$ is the profile of its velocity dispersion, in the radial direction, and $\beta(r)$ is the tracer velocity dispersion anisotropy, which encodes the balance between the tangential and radial components of the velocity dispersion. At a given radius,

$\beta(r)=1-\frac{\sigma_{t}^{2}(r)}{2 \sigma_{r}^{2}(r)}$,

where $\sigma_{t}(r)$ is the tangential component of the velocity dispersion, such that the total (3D) dispersion is given by,

$\sigma_{3 \mathrm{D}}(r)=\sqrt{\sigma_{r}^{2}(r)+\sigma_{t}^{2}(r)}$.

Thus defined, $\sigma_{t}(r)$ includes the contributions to the total velocity dispersion from two mutually orthogonal tangential directions, which are equivalent under the assumption of spherical symmetry.

When applied to a dSph galaxy, for example, equation (2) relates the total mass profile to the spatial distribution and kinematics of stars orbiting in the total gravitational potential (which is dominated by the dark matter). For an observed galaxy, we can measure the projected stellar density, $\Sigma(R)$, and velocity dispersion along the line of sight, ${ }^{3} \sigma_{\text {los }}(R)$. A deprojection is required to map these observable profiles on to the 3D profiles that appear on the right-hand side of equation (2). The projected and 3D stellar density profiles, $\Sigma(R)$ and $\rho(r)$, under the assumption of spherical symmetry, are related by an Abel transform,

$\rho(r)=-\frac{1}{\pi} \int_{r}^{\infty} \frac{\mathrm{d} \Sigma(R)}{\mathrm{d} R} \frac{\mathrm{d} R}{\sqrt{R^{2}-r^{2}}}$.

\footnotetext{
${ }^{2}$ Strictly, $\rho(r)$ is the number density profile of the tracer population, or indeed, the mass (or luminosity) density profile, provided that all tracers have the same mass (or luminosity).

${ }^{3}$ In this paper, a lower case $r$ is used to denote a 3D radius, and an upper case $R$ is used to denote a 2D, projected, radius.
} 
Given only line-of-sight kinematic data for the stars, there exists an inconvenient degeneracy between $\beta(r)$ and $\sigma_{r}(r)$, such that (Binney \& Mamon 1982),

$\Sigma(R) \sigma_{\mathrm{los}}^{2}(R)=2 \int_{R}^{\infty} \rho(r) \sigma_{r}^{2}(r)\left[1-\frac{R^{2}}{r^{2}} \beta(r)\right] \frac{r \mathrm{~d} r}{\sqrt{r^{2}-R^{2}}}$.

In this way, our ignorance of $\beta(r)$ influences both the deprojection of $\sigma_{\text {los }}(R)$ on to $\sigma_{r}(r)$ in equation (6), and the subsequent inference of the enclosed mass profile from equation (2).

In order to make progress, some assumption about the stellar velocity dispersion anisotropy is required, ranging from assuming isotropy $(\beta=0)$, to exploring a wide range of plausible forms for $\beta(r)$ in the fitting procedure (e.g. Wolf et al. 2010).

Through Jeans analysis of eight of the brightest dSphs of the MW, assuming constant $\beta$, Walker et al. $(2009,2010)$ find that the total mass within a sphere of radius equal to the projected stellar half-light radius (effective radius), $R_{\mathrm{e}}$, is relatively well constrained (compared to smaller and larger radii), and seemingly robust against the choice of $\beta$, and of the assumed shape of the total density profile (see also Strigari et al. 2007, 2008; Peñarrubia, McConnachie \& Navarro 2008a,b; Wolf et al. 2010; Amorisco \& Evans 2011). Assuming that the stars follow a Plummer density profile, with a constant and isotropic velocity dispersion, Walker et al. (2009) propose a simple estimator for the total mass enclosed within a sphere of radius $R_{\mathrm{e}}$, which arises immediately from equation (2) given these additional assumptions,

$M\left(<R_{\mathrm{e}}\right)=\frac{5\left\langle\sigma_{\mathrm{los}}\right\rangle^{2} R_{\mathrm{e}}}{2 G}$,

where $\left\langle\sigma_{\text {los }}\right\rangle$ is the (assumed to be constant) line-of-sight stellar velocity dispersion averaged over the whole galaxy. That is, a constraint on the dynamical mass (or equivalently, a point on the circular velocity curve) can be obtained using only the stellar half-light radius and a single value for the stellar velocity dispersion, which does not require spatially resolved kinematic data.

Wolf et al. (2010), on the other hand, argue that the 3D radius within which the sensitivity of the enclosed mass to $\beta$ is minimized is in fact $r_{3}$, the radius at which the logarithmic slope of the stellar density profile, $\mathrm{d} \log \rho(r) / \mathrm{d} \log r$, equals -3 . Assuming that $r_{3}$ is approximately the stellar 3D half-light radius, $r_{\text {half }}$, these authors propose the estimator,

$M\left(<r_{\text {half }}\right)=\frac{3\left\langle\sigma_{\text {los }}\right\rangle^{2} r_{\text {half }}}{G}$,

where they stress that $\left\langle\sigma_{\text {los }}\right\rangle$ must be the luminosity-weighted mean dispersion. This estimator is based on the assumption that $\sigma_{\text {los }}(R)$ remains relatively flat from the centre of the system out to beyond $R_{\mathrm{e}}$, and that $\beta(r)$ does not have an extremum within the stellar distribution. To express equation (8) entirely in terms of observable quantities, Wolf et al. (2010) make the further simplifying assumption that $r_{\text {half }}=4 R_{\mathrm{e}} / 3$, in which case,

$M\left(<\frac{4}{3} R_{\mathrm{e}}\right)=\frac{4\left\langle\sigma_{\mathrm{los}}\right\rangle^{2} R_{\mathrm{e}}}{G}$.

This estimator is of the same form as equation (7), but with $\lambda=4 / 3$ and $\mu=4$, compared to $\lambda=1$ and $\mu=2.5$, in the notation of equation (1). It is worth noting that if both equations (7) and (9) apply, then the enclosed dynamical mass increases by a factor of 1.6 from $R_{\mathrm{e}}$ to $4 R_{\mathrm{e}} / 3$. We test the accuracy of equations (7) and (9) using simulated galaxies in Section 5 , and return to equation (8) in Appendix A.

\section{SIMULATIONS}

We now describe the simulations used in this paper, followed by the definitions employed for subhaloes and galaxies, and the resolution limit we impose to ensure converged galaxy properties.

\subsection{APOSTLE simulations}

The APOSTLE simulations use the 'zoom' technique to resimulate 12 systems consistent with observational constraints on the properties of the Local Group, as described in more detail by Fattahi et al. (2016) and Sawala et al. (2016). The regions were selected from a dark matter only simulation of a cosmologically representative volume of comoving side $100 \mathrm{Mpc}$, using $\Lambda \mathrm{CDM}$ parameters consistent with WMAP7 (Komatsu et al. 2011). The density parameters at redshift zero are $\Omega_{\mathrm{m}}=0.272$ (matter), $\Omega_{\mathrm{b}}=0.0455$ (baryons) and $\Omega_{\Lambda}=1-\Omega_{\mathrm{m}}=0.728$ (cosmological constant). The presentday Hubble parameter is $H_{0}=100 h \mathrm{~km} \mathrm{~s}^{-1} \mathrm{Mpc}^{-1}$, with $h=0.704$. The linear power spectrum is normalized using $\sigma_{8}=0.81$ at redshift zero. The spectral index of primordial fluctuations is $n_{\mathrm{s}}=0.967$. The pairs of MW and M31 analogues were selected on the basis of their separations, relative radial and tangential velocities, and halo masses, along with the recession velocities of outer Local Group members (see Fattahi et al. 2016 for details).

The 12 regions in the APOSTLE suite were simulated using the code from the EAGLE project (Crain et al. 2015; Schaye et al. 2015), at a series of resolution levels, which we label as LR, MR and HR, in order of increasing resolution (low, medium and high). Table 1 lists the dark matter and (initial) gas particle masses in the zoom region at each resolution level, along with the gravitational force softening. All regions have been simulated at LR and $\mathrm{MR}$, and two regions have also been run at HR. The EAGLE code is a version of the smoothed particle hydrodynamics (SPH) code GADGET (Springel 2005) that includes 'subgrid' models for radiative gas cooling and heating (Wiersma, Schaye \& Smith 2009a), reionization, star formation (Schaye 2004; Schaye \& Dalla Vecchia 2008), stellar mass loss and metal enrichment (Wiersma et al. 2009b), stellar feedback (Dalla Vecchia \& Schaye 2012), black hole formation and mergers (Rosas-Guevara et al. 2015), and feedback from active galactic nuclei (Booth \& Schaye 2009). See Crain et al. (2015) and Schaye et al. (2015) for full details of the subgrid models. The hydrodynamics implementation used is ANARCHY (Dalla Vecchia in preparation), which uses the conservative pressure-entropy SPH formulation derived by Hopkins (2013). See Schaller et al. (2015) for a description of ANARCHY and the impact of the changes with respect to the original GADGET SPH scheme on galaxy properties in the EAGLE simulations. The APOSTLE simulations use the 'reference' EAGLE model parameters as described by Schaye et al. (2015).

Table 1. Parameters for each resolution level in the APOSTLE simulations. $m_{\text {DM }}$ and $m_{\text {gas }}$ are the high-resolution (zoom) dark matter and initial gas particle masses, respectively. $\epsilon(z=0)$ is the Plummer-equivalent gravitational force softening at redshift zero. The gravitational force is Newtonian on scales larger than $2.8 \epsilon$. There is a small amount of variation in the particle masses used in different simulations at a given resolution level; average values are quoted here (see Fattahi et al. 2016 for the individual values).

\begin{tabular}{lccc}
\hline Resolution & $m_{\mathrm{DM}}\left(\mathrm{M}_{\odot}\right)$ & $m_{\text {gas }}\left(\mathrm{M}_{\odot}\right)$ & $\epsilon(z=0)(\mathrm{pc})$ \\
\hline LR & $7.1 \times 10^{6}$ & $1.4 \times 10^{6}$ & 710 \\
MR & $5.8 \times 10^{5}$ & $1.2 \times 10^{5}$ & 307 \\
HR & $3.7 \times 10^{4}$ & $7.4 \times 10^{3}$ & 134 \\
\hline
\end{tabular}




\subsection{Halo finding and galaxy definition}

To identify haloes in the simulations, we first make use of the friends-of-friends (FOF) algorithm, considering only dark matter particles, with a linking length of 0.2 times the mean interparticle separation (Davis et al. 1985). Baryonic particles are assigned to the same FOF group as their nearest dark matter particle. Each FOF group is then processed using SUBFIND, which identifies overdense gravitationally self-bound 'subhaloes' (Springel et al. 2001; Dolag et al. 2009). The position of a subhalo is taken to be that of the particle with the minimum value of the gravitational potential. The 'main subhalo' of a FOF group is that whose such particle has the lowest potential in the group. ${ }^{4}$ All other subhaloes are embedded in the main subhalo (a given particle can belong to at most one subhalo). As described by Schaye et al. (2015), we combine any two subhaloes whose separation is less than the stellar half-mass radius of either subhalo, for separations of at most 3 physical kpc. This final adjustment is designed to absorb a very small number of very low mass subhaloes that are dominated by a single baryonic particle of unusually high mass (as a result of exceptional stellar mass loss to a gas particle, or black hole growth).

We define a galaxy to be the set of subhalo star particles within a spherical aperture of radius $r_{\text {gal }}$ equal to 15 per cent of the virial radius, $r_{200}$, as measured from the subhalo centre. ${ }^{5}$ For subhaloes where the actual value of $r_{200}$ is not meaningful, e.g. for the subhalo of a satellite galaxy embedded in a much larger halo, we adopt the value of $r_{200}$ obtained using a relation between $r_{200}$ and the maximum value of the subhalo circular velocity curve, $V_{\max }$, calibrated using main subhaloes in the EAGLE Ref-L025N0752 simulation. ${ }^{6}$ In practice, we make use of the directly measured value of $r_{200}$ when computing $r_{\text {gal }}$ for all main subhaloes, and for any other subhalo for which $r_{200}$ is less than the distance to the furthest subhalo particle. All galaxy properties presented in this paper are computed using the set of subhalo star particles within $r_{\text {gal }}$, with the galaxy centre set to that of its host subhalo. We adopt the rest frame of the centre of mass of the set of star particles defined in this way.

In each simulation volume, we shall refer to the MW and M31 analogues as the 'primary' galaxies. Galaxies within $300 \mathrm{kpc}$ of the centre of either primary galaxy are labelled as 'satellites', and those at larger distances are labelled as 'field' galaxies. This classification is independent of the particular FOF group in which a galaxy resides.

\subsection{Selecting a well-resolved galaxy sample}

We assume that the luminosity of a star particle (which represents a simple stellar population) is proportional to its mass. In this case, $R_{\mathrm{e}}$ is the projected radius that encloses half the stellar mass, as well as half the total stellar luminosity. Fig. 1 shows $R_{\mathrm{e}}$ versus

\footnotetext{
${ }^{4}$ Note that we still refer to this main halo from suBFIND as a subhalo, even though it constitutes the main component of the FOF group. For our purposes, the distinction between the main subhalo and other subhaloes in a FOF group is not important. It is typical in the literature to label the galaxy found in the main subhalo as the 'central' galaxy of the FOF group, while other subhaloes host 'satellite' galaxies. However, this nomenclature can be confusing in some cases, e.g. in the APOSTLE simulations the MW and M31 analogues can reside in the same FOF group.

${ }^{5} r_{200}$ is the radius of the sphere that encloses a mean density equal to 200 times the critical density of the Universe.

${ }^{6}$ Ref-L025N0752 has the highest resolution available in the EAGLE simulation suite, which is similar to that of the MR APOSTLE simulations. The assumed cosmological parameters are slightly different to those used in the APOSTLE simulations, which is not important for our purposes.
}

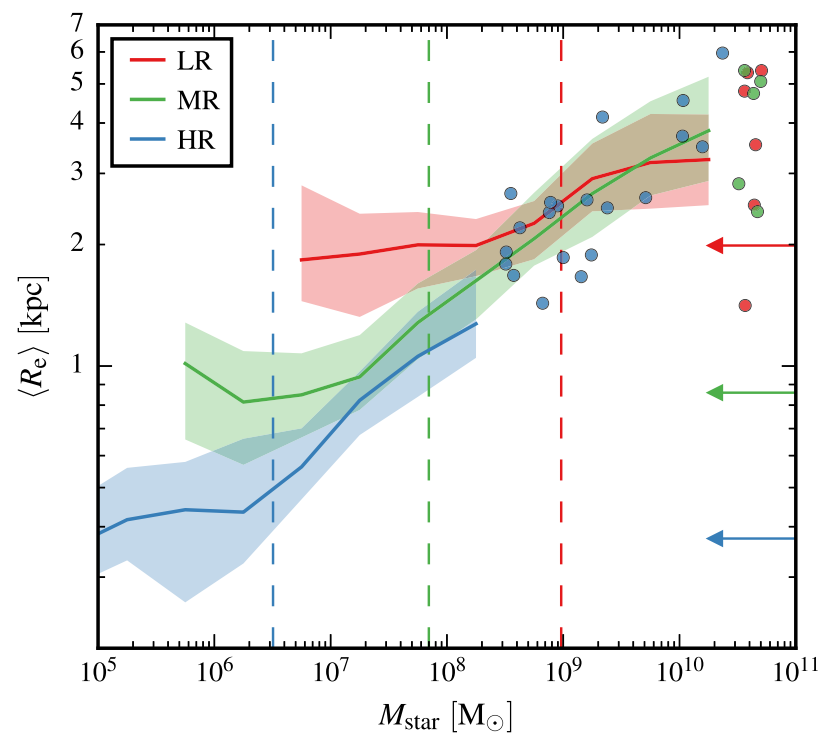

Figure 1. Mean projected stellar half-mass radius, $\left\langle R_{\mathrm{e}}\right\rangle$, versus stellar mass, $M_{\text {star }}$, for all galaxies that have at least 10 star particles in the APOSTLE simulations at each resolution level (12 LR, 12 MR and 2 HR simulations; different colours). The $\left\langle R_{\mathrm{e}}\right\rangle$ values are averaged over 1536 evenly distributed projections. For each resolution, the median is shown as a solid line, and the 16th-84th percentiles are indicated by a shaded region of the same colour. Points are shown instead for bins with fewer than 10 galaxies. The vertical dashed lines show the minimum $M_{\text {star }}$ of the set of galaxies composed of at least 1000 star particles at each resolution. The horizontal arrows indicate 2.8 times the gravitational softening at each resolution (see Table 1).

stellar mass, $M_{\text {star }}$, for all galaxies in the APOSTLE simulations that are composed of at least 10 star particles, split by resolution level. The $R_{\mathrm{e}}$ values are averaged from projecting over 1536 evenly distributed lines of sight for each galaxy, making use of the HEALPIX spherical tessellation (Górski et al. 2005). ${ }^{7}$ This set of sightlines will be exploited throughout this paper.

In the following, we consider all galaxies in the highest resolution realization of each of the 12 APOSTLE regions (10 MR and $2 \mathrm{HR}$ simulations) that are resolved with at least 1000 star particles. This conservative threshold has been chosen so that the stellar mass-size relation is converged at each resolution level (see also Schaye et al. 2015). The vertical lines in Fig. 1 show the minimum $M_{\text {star }}$ of the set of galaxies with at least 1000 star particles at each resolution. Note that the masses of star particles vary according to the level of enrichment of the gas from which they formed, and the extent of their own mass-loss since their birth. There is also a small amount of variation in the initial gas particle mass (and dark matter particle mass) between different simulations at each resolution (see Table 1).

\section{GENERAL GALAXY PROPERTIES}

In this section, we select a sample of simulated galaxies whose stellar kinematics is dominated by dispersion. We then investigate the basic properties of the galaxies in order to assess how realistic they are. We present radial profiles of the projected stellar density and line-of-sight velocity dispersion for galaxies that are resolved at the highest resolution level, along with profiles of their stellar velocity dispersion anisotropy. These profiles are central to the mapping between observables and the total 3D mass profile

\footnotetext{
${ }^{7}$ This corresponds to 3072 HEALPIX pixels.
} 


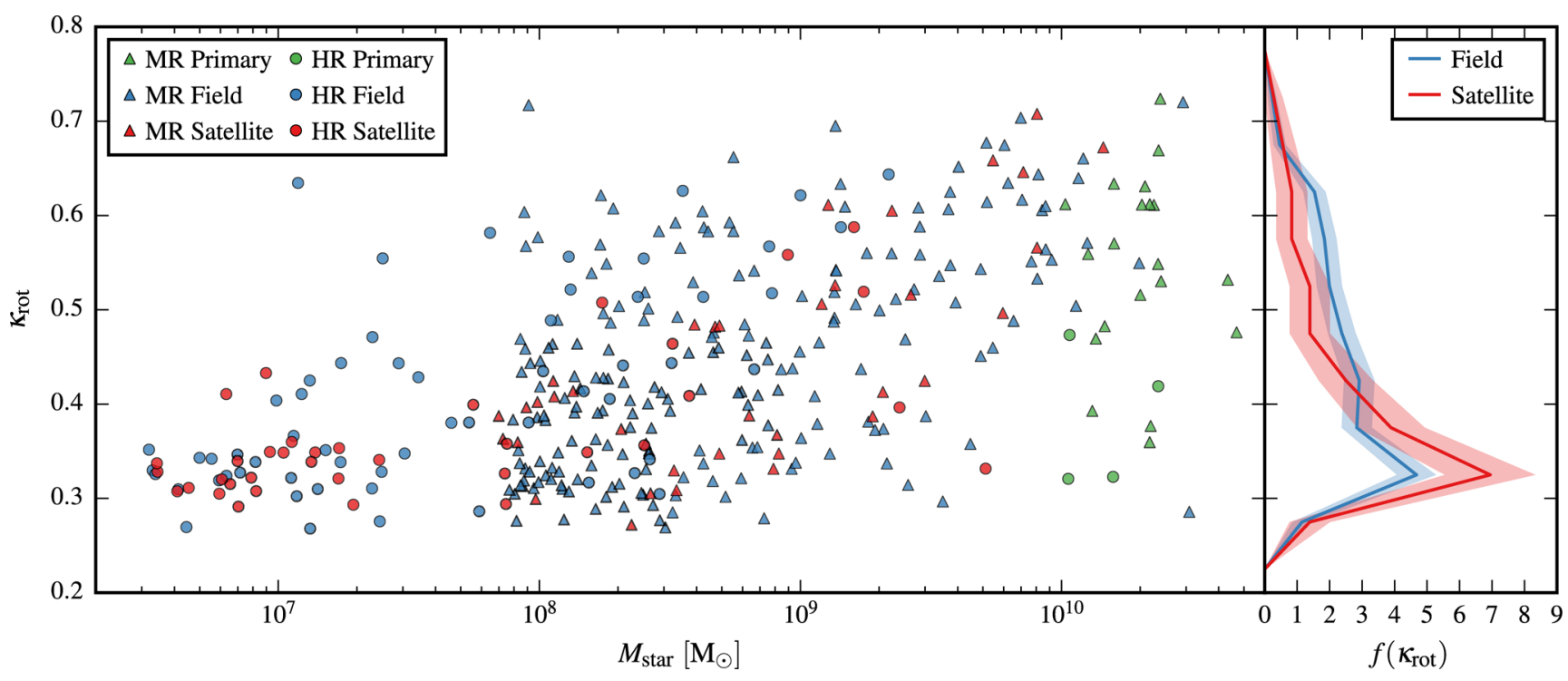

Figure 2. Kinematic measure, $\kappa_{\text {rot }}$, used to discriminate amongst galaxies with different levels of stellar rotational support (see equation 10), shown versus stellar mass, $M_{\text {star }}$, for all galaxies in our simulated sample. The galaxies are classified according to whether they are one of the MW or M31 analogues (primary), within $300 \mathrm{kpc}$ of either of these (satellite), or located at larger distances (field). The symbol shapes indicate the resolution level at which each galaxy has been simulated (MR or HR). Our dispersion-dominated galaxy sample consists of all galaxies that have $\kappa_{\text {rot }}<0.5$. The panel on the right shows the distributions, $f\left(\kappa_{\text {rot }}\right)$, of $\kappa_{\text {rot }}$ for all field and satellite galaxies. Each distribution is normalized to have unit area. The shaded regions show the $1 \sigma$ error due to the Poisson noise on each bin count.

(see equations 2, 5 and 6), and thus represent the basic dynamical quantities that enter into the Jeans analysis. We then place the simulated galaxies in the context of the Local Group by comparing their stellar masses, projected half-mass radii and velocity dispersions with observational data. Finally, we investigate the degree to which the simulated galaxies are spherically symmetric and in dynamical equilibrium.

\subsection{Balance between dispersion and rotational support}

A key assumption underpinning the spherical Jeans equation is that the system under consideration is supported against gravitational collapse by dispersion, rather than rotational or other streaming motion. This clearly is not the case for galaxies with prominent stellar discs. To identify systems with significant rotational support, we make use of the quantity $\kappa_{\text {rot }}$ as introduced by Sales et al. (2012), which they define as 'the fraction of kinetic energy invested in ordered rotation'. $\kappa_{\text {rot }}$ is computed as

$\kappa_{\mathrm{rot}}=\frac{1}{K_{\mathrm{star}}} \sum_{i} \frac{m_{i}}{2}\left(\frac{j_{z, i}}{R_{x y, i}}\right)^{2}$,

where $K_{\text {star }}$ is the total stellar kinetic energy. For each star particle, $i$, of mass $m_{i}, j_{z, i}$ is the component of its specific angular momentum in the direction of the total stellar angular momentum vector, $\boldsymbol{L}_{\mathrm{star}}$, and $R_{x y, i}$ is its distance from the axis ( $z$ ) defined by $\boldsymbol{L}_{\text {star }}$.

Strictly speaking, $\kappa_{\text {rot }}$ is not directly sensitive to ordered rotation, because the sign of the rotation about the $z$-axis is lost in the squared term in equation (10). Yet systems that exhibit strong rotation have high values of $\kappa_{\text {rot }}$, which makes $\kappa_{\text {rot }}$ a useful measure to discriminate between systems that are dispersion dominated and those dominated by rotating discs. A pure disc galaxy with stars on perfect circular orbits would have $\kappa_{\text {rot }}=1$, while instead $\kappa_{\text {rot }}=1 / 3$ for a dispersion-supported system with isotropic orbits (since $K_{\text {star }}$ includes all three orthogonal velocity components, but the summation in equation 10 considers only one such component).
Thus, $\kappa_{\text {rot }}$ decreases from unity as random motion becomes more important, with a lower limit in the region of $\kappa_{\text {rot }} \sim 1 / 3$. However, dispersion-supported systems with radially biased orbits can have $\kappa_{\text {rot }}<1 / 3$, since less of the kinetic energy is invested in tangential motion than in the isotropic case. Similarly, tangentially biased orbits imply $\kappa_{\text {rot }}>1 / 3$.

Fig. 2 shows $\kappa_{\text {rot }}$ versus $M_{\text {star }}$ for all galaxies in our sample (as defined in Section 3.3). It can be seen that the simulations predict a broad range of stellar morphologies, according to this kinematic measure. For $M_{\text {star }} \gtrsim 10^{9} \mathrm{M}_{\odot}$, rotation-dominated galaxies $\left(\kappa_{\text {rot }}>0.5\right)$ are slightly more prevalent than those that are dominated by dispersion $\left(\kappa_{\text {rot }}<0.5\right)$. However, at progressively lower stellar masses, the galaxies are progressively more likely to be dispersion dominated. For $M_{\text {star }} \lesssim 10^{8} \mathrm{M}_{\odot}$, only a small fraction of galaxies have significant levels of rotational support. The distributions of $\kappa_{\text {rot }}$ for satellites and field galaxies are not significantly different. The MR and HR simulations predict similar distributions of $\kappa_{\text {rot }}$ at a given $M_{\text {star }}$, over the common mass range explored.

In this paper, our main focus is on galaxies with dispersiondominated stellar kinematics, i.e. those with $\kappa_{\text {rot }}<0.5$. The galaxy sample defined in this way includes 70 per cent of our full sample.

\subsection{Stellar density and kinematic profiles}

Projected stellar density profiles, $\Sigma(R)$, for dispersion-dominated field and satellite galaxies in the HR simulations are shown in Fig. 3, split into bins of stellar mass. The profiles are obtained by projecting over a large number of lines of sight (as described in Section 3.3), and are stacked for clarity, showing field and satellite galaxies separately. There is no significant difference between the profiles for field and satellite galaxies in any mass bin. There is a small variation in the shape of the profiles with increasing stellar mass, such that the stellar density is more centrally concentrated in the highest mass bin than in the other bins (see dashed line that repeats the median profile from the lowest mass bin). Projected stellar number density 


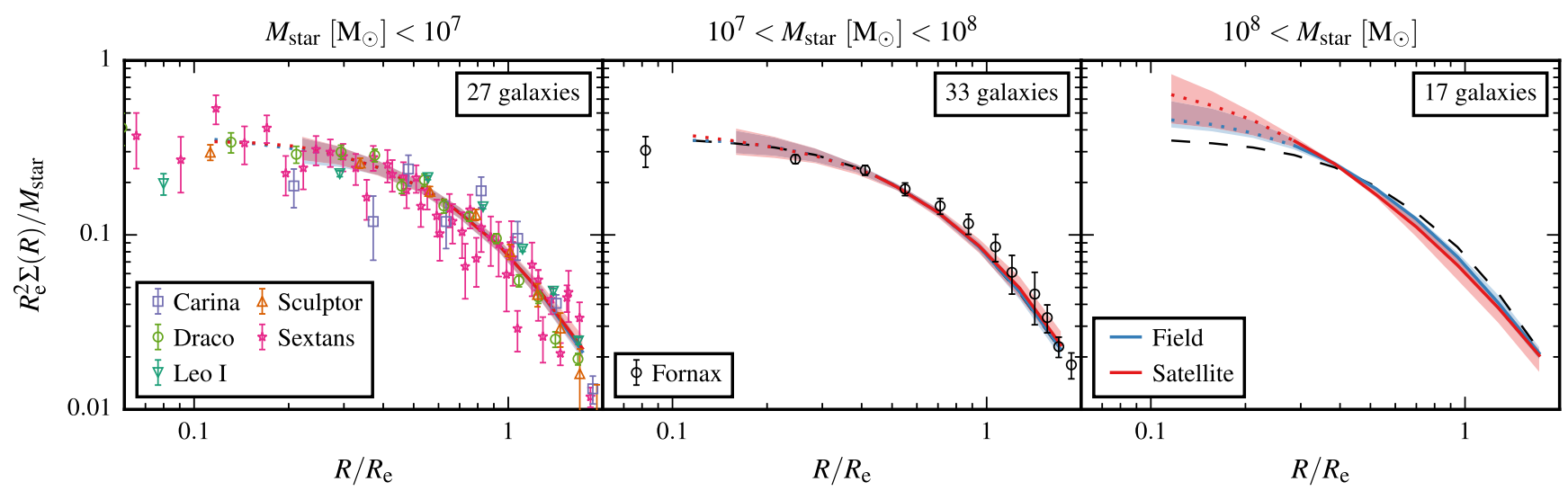

Figure 3. Projected stellar density profiles, $\Sigma(R)$, for dispersion-dominated $\left(\kappa_{\mathrm{rot}}<0.5\right)$ field and satellite galaxies in the HR simulations. The solid lines are the median projected profiles obtained from 1536 evenly distributed projections for all galaxies of a given type (different colours; legend in the right-hand panel), around which the shaded regions of the same colour indicate the 16th-84th percentile spread. Each individual profile is scaled, prior to stacking, by the projected stellar half-mass radius, $R_{\mathrm{e}}$, for that line of sight, and the stellar mass, $M_{\mathrm{star}}$. The shaded regions are not shown below the gravitational softening (see Table 1; in units of the median $R_{\mathrm{e}}$ in each panel), and the median profiles are dotted below 2.8 times the softening. The panels show different stellar mass ranges, and are labelled with the number of simulated galaxies they contain. As a visual aid, the median profile for all galaxies in the lowest mass bin is repeated as a dashed black line in the higher mass bins. The symbols with error bars show data for dSph satellites of the MW, where the data for each dSph are plotted in the relevant panel for its stellar mass following McConnachie (2012). The data shown are for Carina (Muñoz et al. 2006), Draco (Odenkirchen et al. 2001), Fornax (Coleman et al. 2005), Leo I (Smolčić et al. 2007), Sculptor (Battaglia et al. 2008) and Sextans (Irwin \& Hatzidimitriou 1995). The measurements are scaled in the same way as the simulation predictions, assuming either a Gaussian (Leo I) or Plummer (all others) density profile fit.

profile data for bright dSphs of the MW are shown alongside the simulation predictions. The measured profiles have been rescaled assuming the best fitting of either a Plummer or Gaussian density profile model (with $M_{\text {star }}$ and $R_{\mathrm{e}}$ as free parameters). We find that the data points for Leo I are best described by a Gaussian profile, while the other galaxies shown each prefer a Plummer profile. The simulation predictions closely trace the observational data, in which the degree of scatter varies for each dSph. Thus, we can see that the simulated galaxies have realistic stellar density distributions.

Line-of-sight velocity dispersion profiles, $\sigma_{\text {los }}(R)$, are shown in Fig. 4 for the same set of simulated galaxies. ${ }^{8}$ These profiles show each galaxy individually, using the median result from all lines of sight. The profiles tend to be quite flat with radius. The typical dispersions and radial extents of the galaxies scale closely with $M_{\text {star }}$, and there are two particularly large satellites with dispersions in excess of $\sim 50 \mathrm{~km} \mathrm{~s}^{-1}$ (note that there are two HR simulations, and hence four primary galaxies, which between them host the satellites shown). Recall that an assumption used in the derivation of each of the mass estimators discussed in Section 2 is that $\sigma_{\text {los }}(R)$ is relatively flat (or even constant). To see the typical profile shapes more clearly, and to identify if there are systematic differences in $\sigma_{\text {los }}(R)$ for field and satellite galaxies, we show scaled versions of these profiles in Fig. 5, averaging over all lines of sight in our standard set. While the scatter in the profiles for both types of galaxy is large, such that there is no significant difference between the two populations, the median profiles are somewhat flatter for field than for satellite galaxies, in the two smaller mass intervals. The satellites in these panels tend to have relatively high velocity dispersions in their centres, and relatively low dispersions in their outer parts, compared with field galaxies. The median profiles intersect near the projected half-mass radius. The data points in Fig. 5 show profiles for bright dSphs of

\footnotetext{
${ }^{8}$ When computing velocity dispersions, we weight by the particle mass For example, the squared velocity dispersion in the $x$ direction is given by $\sigma_{x}^{2}=\left(\sum_{i} m_{i} v_{x, i}^{2}\right) /\left(\sum_{i} m_{i}\right)$, where each particle, $i$, has mass $m_{i}$, and velocity $v_{x, i}$ in the $x$ direction.
}

the MW (Strigari et al. 2010). The simulated galaxies reproduce the flatness in $\sigma_{\text {los }}(R)$ observed for the dSphs.

Fig. 6 shows spherically averaged profiles of the velocity dispersion anisotropy, $\beta(r)$, defined in equation (3), again examining the highest resolution dispersion-dominated field and satellite galaxies, split by stellar mass. The galaxies predominantly have radially biased dispersions $(\beta>0)$, and only a small number of galaxies, which are in the most massive subset, have $\beta \lesssim-0.2$ at any radius. The profiles are similar in form for field galaxies and the satellites, and there are no obvious distinctions between the two populations, except that in the lowest mass bin the field galaxies tend to be slightly more radially biased than the satellites, at both small and large radii. The anisotropy introduces a key degeneracy in Jeans analysis (see Section 2), and the mass estimators of interest here assume either that $\beta(r)$ is monotonic, or indeed that $\beta$ has a negligible impact on the recovered mass, and so can be assumed to equal zero.

\subsection{Galaxy sizes and integrated dispersions}

The dynamical mass estimators described in Section 2 use only two measurements: the projected stellar half-light radius and the line-of-sight stellar velocity dispersion. In Fig. 7, we plot these two quantities against stellar mass for all galaxies in our sample. The simulations predict a relatively tight relationship between $\left\langle\sigma_{\text {los }}\right\rangle$ and $M_{\text {star }}$, while the relationship between $R_{\mathrm{e}}$ and $M_{\text {star }}$ exhibits a somewhat higher level of scatter. There is clear convergence between the results from the two resolution levels, which for the stellar masssize relation reflects our chosen threshold of 1000 star particles (see Section 3.3). There is no obvious difference between the velocity dispersions or sizes of field and satellite galaxies at a given stellar mass.

Observational data for galaxies within $3 \mathrm{Mpc}$ of the Sun as compiled by McConnachie (2012) are shown alongside the simulation predictions in Fig. 7. Given our conservative limit on the number of star particles required for a galaxy to be included in our sample, we consider simulated galaxies down to stellar masses close to that of Sculptor, although the simulations do contain galaxies 

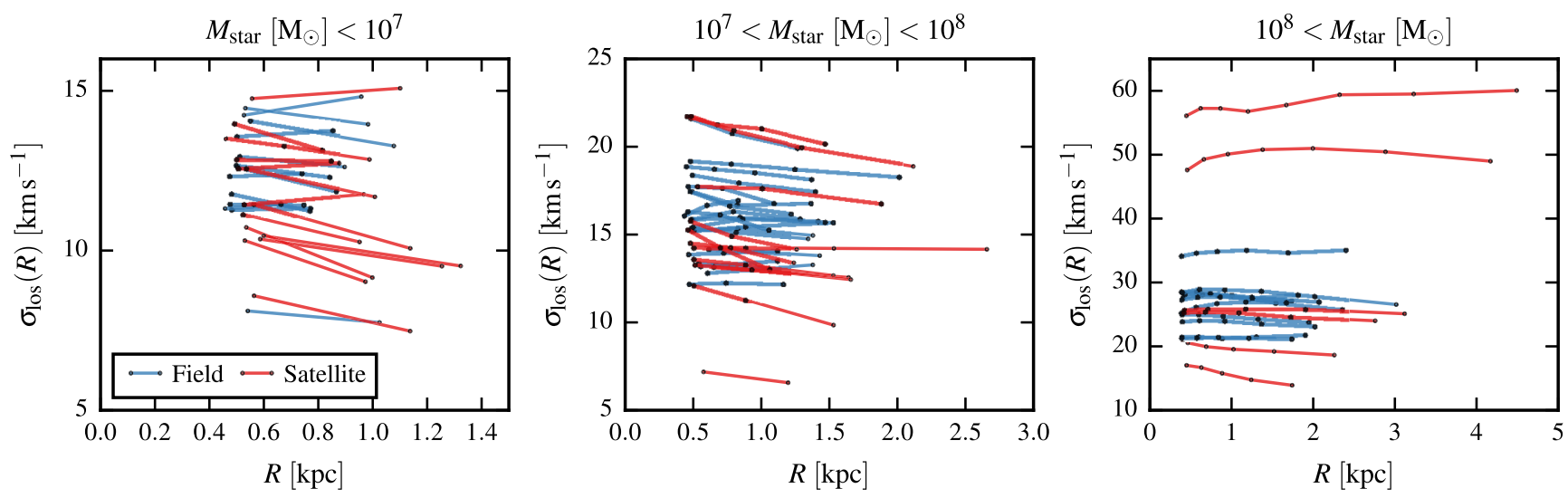

Figure 4. Line-of-sight stellar velocity dispersion profiles, $\sigma_{\operatorname{los}}(R)$, for dispersion-dominated $\left(\kappa_{\text {rot }}<0.5\right)$ field and satellite galaxies in the HR simulations, where each panel shows a different stellar mass range. The line shown for each galaxy is the median profile obtained from projecting over 1536 evenly distributed lines of sight, considering star particles at projected radii between 2.8 times the gravitational softening (see Table 1) and twice the mean projected stellar half-mass radius of the galaxy. The bin edges are evenly spaced in the logarithm of $R$, and the points shown are the linear means of the bin edges. The number of bins used for each galaxy is a function of its star particle count. Note that the axis limits are different for each panel.

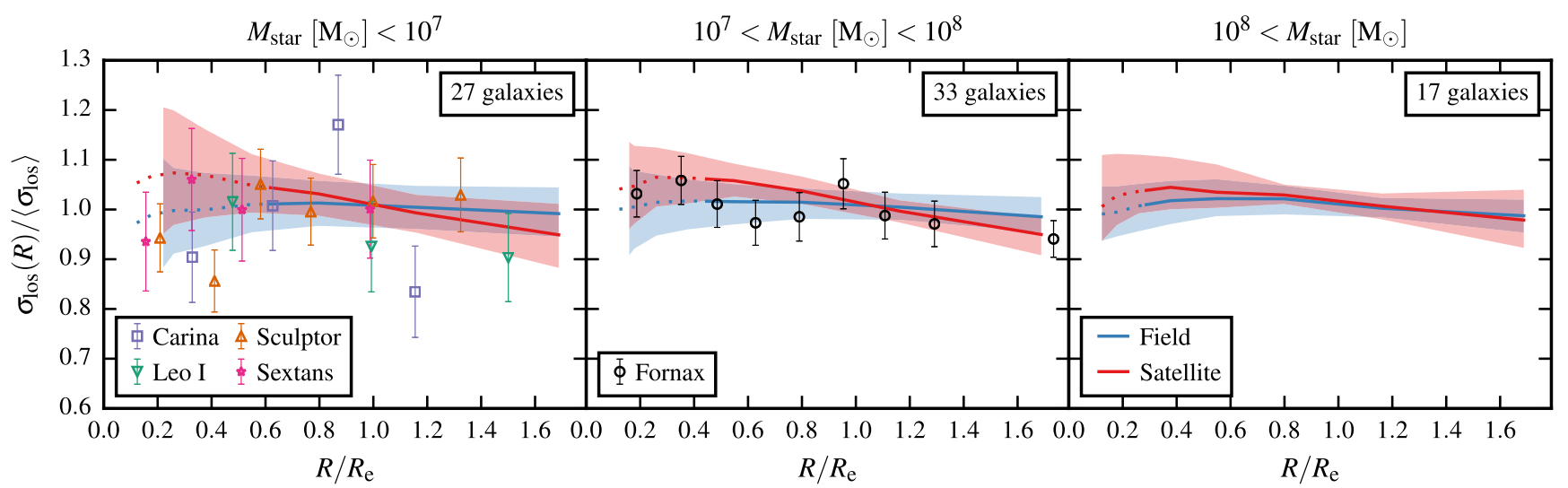

Figure 5. Line-of-sight stellar velocity dispersion profiles, $\sigma_{\text {los }}(R)$, for dispersion-dominated $\left(\kappa_{\text {rot }}<0.5\right)$ field and satellite galaxies in the HR simulations, where each panel shows a different stellar mass range (labelled with the number of galaxies). The solid lines are the median profiles obtained from 1536 evenly distributed projections for all galaxies of a given type (different colours; legend in the right-hand panel), around which the shaded regions of the same colour indicate the 16th-84th percentile spread. Each individual profile is scaled, prior to stacking, by the projected stellar half-mass radius, $R_{\mathrm{e}}$, and the mean stellar velocity dispersion of the whole galaxy, $\left\langle\sigma_{\text {los }}\right\rangle$, for that line of sight. The shaded regions are not shown below the gravitational softening (see Table 1; in units of the median $R_{\mathrm{e}}$ in each panel), and the median profiles are dotted below 2.8 times the softening. The symbols with error bars show data for dSph satellites of the MW, where the data for each $\mathrm{dSph}$ are plotted in the relevant panel for its stellar mass following McConnachie (2012). The profiles shown are for Carina, Fornax, Leo I, Sculptor and Sextans, as computed by Strigari, Frenk \& White (2010). The measurements are scaled in the same way as the simulation predictions, using the half-light radii from McConnachie (2012).

as faint as Draco. The simulation predictions are consistent with the observational constraints, over the large range in stellar mass shown.

It is apparent in Fig. 7 that the simulations predict somewhat less scatter in size at fixed stellar mass than seen in the observational data. The majority of observed galaxies, including the Magellanic Clouds and Fornax, lie on the predicted relation but a substantial fraction are smaller, for their stellar mass, than the smallest galaxies in the simulations. As shown in Fig. 1, at each resolution level, the stellar mass-size relation flattens out at low masses, and the minimum size scales in the same way as the gravitational softening (the horizontal arrows show the scale above which the force is Newtonian, at each resolution). It thus appears that the resolution in the simulations is not quite high enough to account for the sizes of all the observed galaxies that have stellar masses close to those of the smallest resolved galaxies in the simulations. However, for our purpose, which is to test the validity of dynamical mass estimators using the simulated galaxies, it does not matter if resolution effects have had a marginal impact on the galaxy sizes, provided that the galaxies have self-consistent internal dynamics.

\subsection{Equilibrium and spherical symmetry}

The spherical Jeans equation applies to spherically symmetric systems that are in equilibrium. It is reasonable to expect that simple mass estimators based on this equation will fail for galaxies that deviate significantly from either of these assumptions.

In order to quantify the dynamical state of a galaxy in our sample, we consider the ratio of the total kinetic and gravitational potential energies of its star particles,

$\gamma=\frac{2 K_{\text {star }}}{U_{\text {star }}}$, 


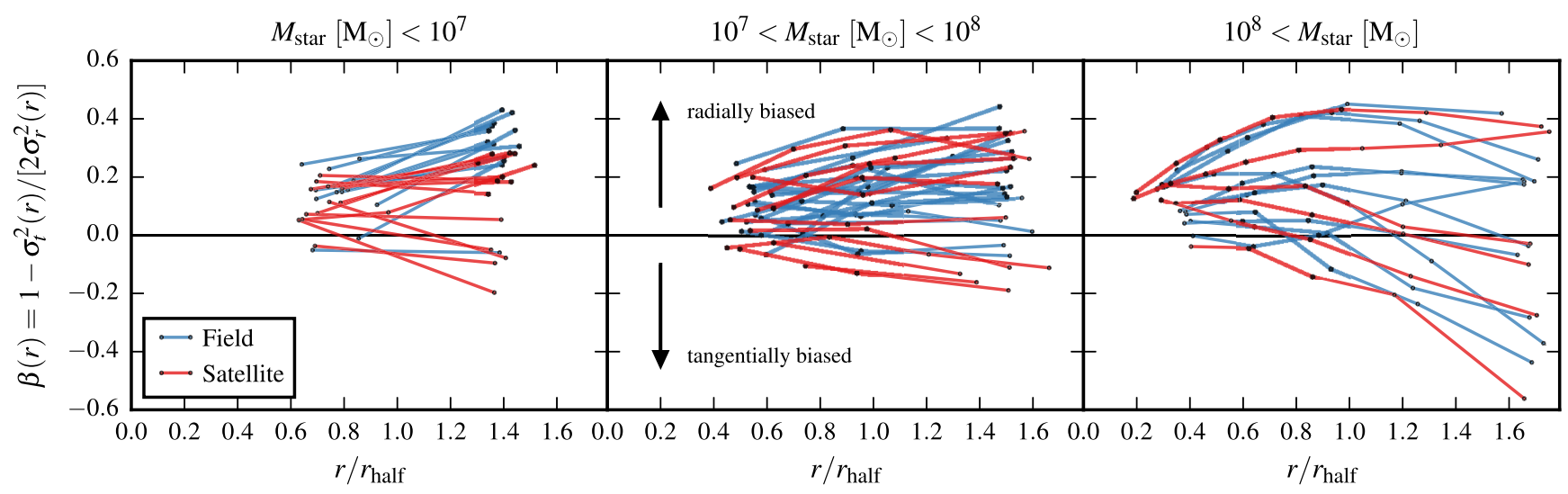

Figure 6. Spherically averaged stellar velocity dispersion anisotropy profiles, $\beta(r)$, for dispersion-dominated $\left(\kappa_{\text {rot }}<0.5\right)$ field and satellite galaxies in the HR simulations. $\beta(r)$ is negative for tangentially biased dispersions, zero for isotropy and positive for radially biased dispersions (see equation 3 ). The panels show different stellar mass ranges, as labelled. The profiles include all star particles between a radius of 2.8 times the softening (see Table 1) and twice the 3D stellar half-mass radius, $r_{\text {half }}$. The bin edges are evenly spaced percentiles of the radial distribution of the star particles, and the points show the median galactocentric radius of the star particles in each bin. The number of bins used for a given galaxy depends on how many star particles it has.

where $K_{\text {star }}$ is the total stellar kinetic energy, and $U_{\text {star }}$ is the sum of the gravitational potential energy of each star particle due to the full mass distribution of the subhalo to which the star particles of the galaxy belong. In computing $U_{\text {star }}$, we shift the zero-point of the potential to coincide with the galactic centre. Equation (11) is similar in appearance to the virial ratio for an isolated system (such that $\gamma$ would equal 1 if the stars were an isolated self-gravitating system in equilibrium, with the potential taken to be zero at an infinite distance from the system). However, $\gamma$ refers only to the stellar component, not to the whole dynamical system, and we measure the potential energy with respect to the bottom of the potential well. There is no natural $\gamma$ value for equilibrium systems, and in the case of equilibrium the value of $\gamma$ still depends on the form of the gravitational potential. The upper panel of Fig. 8 shows $\gamma$ versus $M_{\text {star }}$ for all galaxies in our sample. We consider a galaxy to be out of equilibrium if it has a $\gamma$ value substantially higher or lower than the median value for its stellar mass (black line). We define $\tilde{\gamma}$ to be the ratio of $\gamma$ to the median line shown for each galaxy. Note that the trend in $\gamma$ observed as a function of $M_{\text {star }}$ in Fig. 8 does not imply a trend towards or away from equilibrium.

In order to quantify how close to spherically symmetric a galaxy is, we compute its stellar sphericity, $s$, as follows. We first compute the reduced inertia tensor, $\mathbf{I}$, of the star particles, which projects the mass distribution on to a unit sphere, so that the shape determination does not depend on the radial distribution of the particles (e.g. Bett 2012). This symmetric tensor has components $I_{i j}$, where $i, j \in\{1,2$, 3 such that,

$I_{i j}=\frac{1}{M_{\mathrm{star}}} \sum_{n} m_{n} \frac{r_{n, i} r_{n, j}}{r_{n}^{2}}$,

where each star particle, $n$, has mass $m_{n}$ and is located at a distance $r_{n}$ from the galactic centre. $r_{n, i}$ and $r_{n, j}$ are the coordinates of the star particle with respect to the galactic centre, in the $i$ and $j$ directions, respectively. Taking the eigenvectors of $\mathbf{I}$ to be the principal axes of an ellipsoid, with axis lengths $a \geq b \geq c$ given by the square roots of the eigenvalues of $\mathbf{I}$, the sphericity is $s=c / a$ (a sphere has $s=1$ ). The lower panel of Fig. 8 shows $s$ versus stellar mass for all galaxies in our sample. Naturally, the rotation-dominated galaxies (unfilled symbols) tend be more flattened (and thus have lower $s$ ) than those that are dispersion dominated (filled symbols). However, there are some galaxies that are dispersion dominated according to our kinematic measure $\left(\kappa_{\text {rot }}\right)$ that have lower sphericity than the vast majority of rotation-dominated galaxies. Note that $s$ does not distinguish between disc-like flattening and elongation, as this measure takes into account only two of the axes. The relationship between $s$ and $\kappa_{\text {rot }}$ has a large amount of scatter, although the scatter decreases (and $s$ decreases) with increasing $\kappa_{\text {rot }}$. Looking at Fig. 8, it appears that satellites are preferentially closer to spherical than field galaxies (see distributions in the right-hand panel, which consider only the dispersion-dominated galaxies). This effect is consistent with the expectation for satellite systems that have been tidally stripped (Barber et al. 2015). The sphericity also increases on average as stellar mass decreases in Fig. 8.

Given the broad range of 3D shapes and the spread in energy ratios seen for the simulated galaxies in Fig. 8, there clearly exist galaxies within our (dispersion-dominated, or indeed, full) sample for which the assumptions of spherical symmetry and equilibrium are strongly violated. In the following, it will be particularly interesting to see how the simple mass estimators perform when applied to such galaxies.

\section{ACCURACY OF MASS ESTIMATORS}

Having demonstrated that the galaxies in our simulations have realistic projected stellar density and velocity dispersion distributions, with a range of 3D shapes, velocity dispersion anisotropies, levels of dispersion support and departures from dynamical equilibrium, we now apply the simple mass estimators proposed by Walker et al. (2009) and Wolf et al. (2010) in order to assess their accuracy as a function of various galaxy properties.

For convenience, let us denote the ratio of the estimated mass to the true dynamical mass within some sphere as $\alpha$. Specifically, for the estimator of Walker et al. (2009), from equation (7),

$\alpha_{\text {Walker }}=\frac{5\left\langle\sigma_{\mathrm{los}}\right\rangle^{2} R_{\mathrm{e}}}{2 G M\left(<R_{\mathrm{e}}\right)}$,

and, for the estimator of Wolf et al. (2010), from equation (9),

$\alpha_{\text {Wolf }}=\frac{4\left\langle\sigma_{\mathrm{los}}\right\rangle^{2} R_{\mathrm{e}}}{G M\left(<4 R_{\mathrm{e}} / 3\right)}$.

We emphasize that in both equations (13) and (14), the denominator is the total mass within a sphere of radius proportional to the value of 


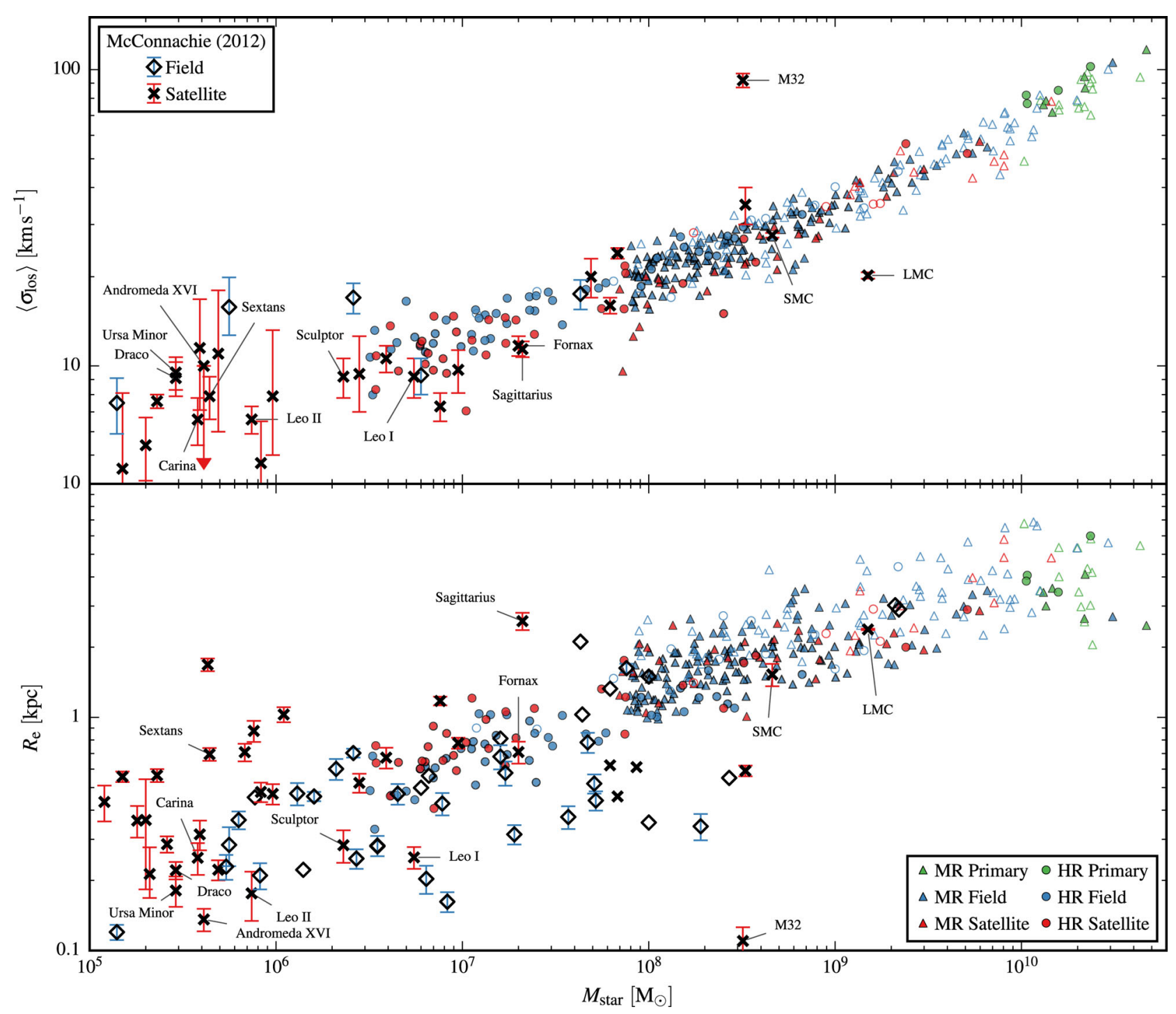

Figure 7. Line-of-sight stellar velocity dispersion, $\left\langle\sigma_{\text {los }}\right\rangle$ (upper), and projected stellar half-mass radius, $R_{\mathrm{e}}$ (lower), versus stellar mass, $M_{\text {star }}$, for all galaxies in our simulated sample. The $\left\langle\sigma_{\mathrm{los}}\right\rangle$ and $R_{\mathrm{e}}$ values have been computed for a single, randomly chosen, line of sight through the simulations. The galaxies are classified according to whether they are one of the MW or M31 analogues (primary), within $300 \mathrm{kpc}$ of either of these (satellite), or at larger distances (field), as indicated in the lower right legend (different colours). The symbol shapes indicate the resolution level at which the galaxies have been simulated (MR or HR). Dispersion-dominated galaxies $\left(\kappa_{\text {rot }}<0.5\right)$ are plotted as filled symbols, while rotation-dominated galaxies $\left(\kappa_{\text {rot }}>0.5\right)$ are plotted as unfilled symbols of the same colour and shape. Data compiled by McConnachie (2012) for galaxies in this stellar mass range within 3 Mpc of the Sun (excluding the MW and M31) are shown alongside the simulation predictions (upper left legend). The observed galaxies are categorized as satellites if they are associated with the MW or M31, otherwise they are classed as field galaxies. The error bars indicate the published uncertainties on the radii and velocity dispersions (where available). We supplement the McConnachie (2012) data with half-light radii for the Small Magellanic Cloud and the Large Magellanic Cloud from Subramanian \& Subramaniam (2012) and Weinberg \& Nikolaev (2001, disc model with no bar), respectively, integrating the published exponential density profile fits in each case. The labelled points are the 11 'classical' satellites of the MW, along with M32 (which has an exceptionally large dispersion and small size for its mass), and Andromeda XVI (dispersion value is upper limit; downward arrow). We assume that the luminosity of a star particle is proportional to its mass, and therefore $R_{\mathrm{e}}$ is both the half-mass and half-light radius for the simulated galaxies.

$R_{\mathrm{e}}$ obtained for a given line of sight. For each galaxy in our sample, we measure $R_{\mathrm{e}},\left\langle\sigma_{\text {los }}\right\rangle, M\left(<R_{\mathrm{e}}\right)$ and $M\left(<4 R_{\mathrm{e}} / 3\right)$ for 1536 evenly distributed lines of sight. The masses are obtained from all subhalo particles within the relevant radius, including the contributions from dark matter, gas, stars and black holes, where present.

\subsection{Dispersion support}

The mass estimators assume that the dynamical system is supported by dispersion. We begin by applying the estimators to all galaxies in our sample, including those with prominent stellar discs (for which we would not expect an observer to use such an estimator). Fig. 9 shows the distributions of $\alpha$ values obtained by applying both estimators to all projections of every galaxy, split according to whether the stellar motions within the galaxy are dominated by dispersion or rotation (see Section 4.1).

For both kinematic regimes, each estimator has a bias in the median of no more than 5 per cent (dashed lines), accompanied by a large scatter (see dotted lines and standard deviation values). It is interesting that there is no dramatic change in the accuracy of 

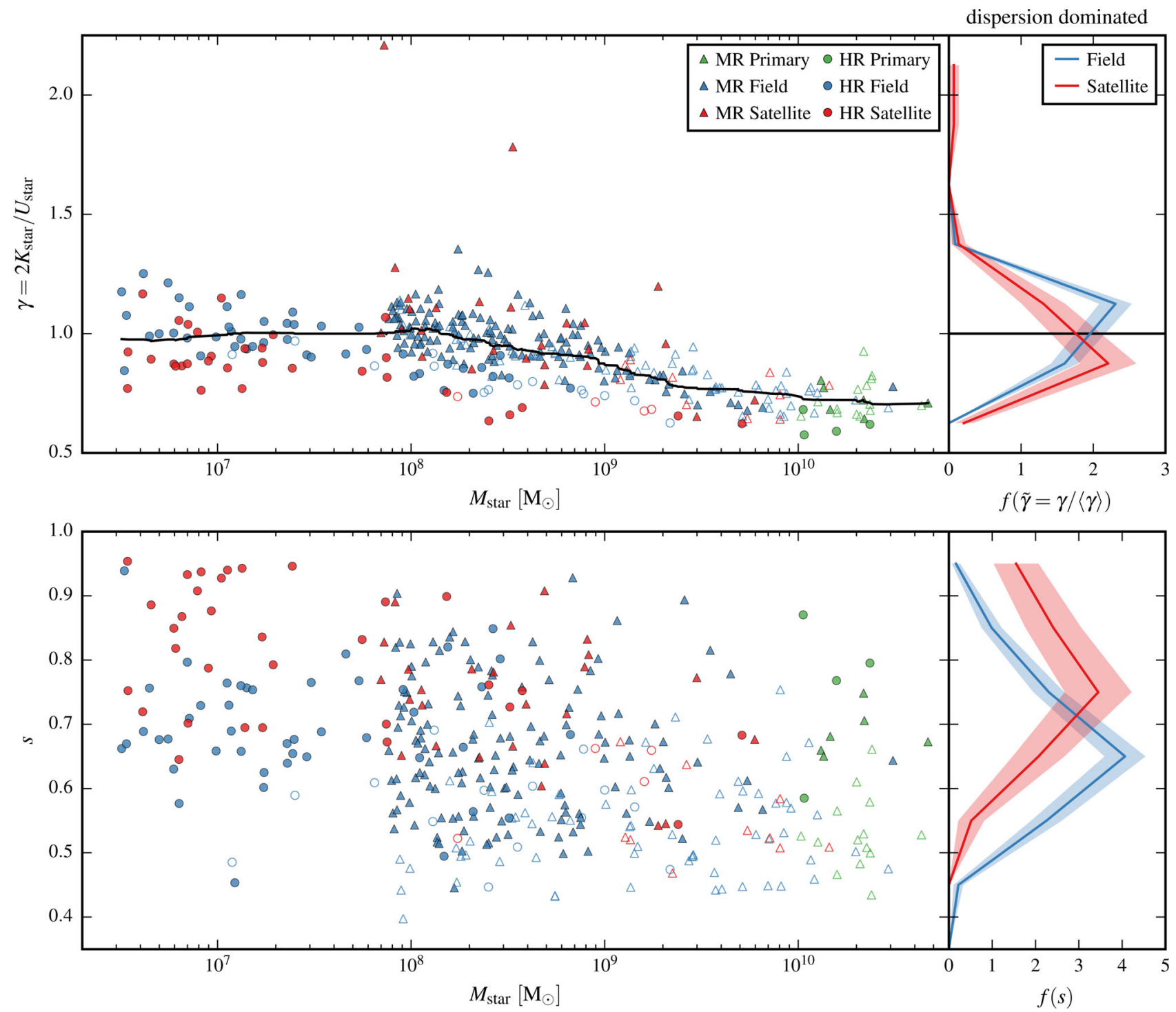

Figure 8. Stellar energy ratio, $\gamma$ (upper; see equation 11), and stellar sphericity, $s$ (lower; derived from the reduced inertia tensor as defined in equation 12), versus stellar mass, $M_{\text {star }}$, for all galaxies in our simulated sample. The colours show whether a galaxy is one of the MW or M31 analogues (primary), within $300 \mathrm{kpc}$ of either of these (satellite), or at larger distances (field). The symbol shapes indicate the resolution level (MR or HR). Dispersion-dominated galaxies $\left(\kappa_{\text {rot }}<0.5\right)$ are plotted as filled symbols, while the symbols are unfilled for rotation-dominated galaxies $\left(\kappa_{\text {rot }}>0.5\right)$. The black line in the upper panel shows the running median, $\langle\gamma\rangle$, of the $\gamma$ values, considering at most 100 neighbouring galaxies in $M_{\text {star }}$ for each galaxy. We define $\tilde{\gamma}$ as the ratio of $\gamma$ to this median line for each galaxy, and assume that galaxies with $\tilde{\gamma} \approx 1$ are in equilibrium. The panels on the right show the distributions of the rescaled energy ratio, $f(\tilde{\gamma})$ (not the distribution of $\gamma$ itself), and the sphericity, $f(s)$, on the same vertical scales as the main panels, for only the dispersion-dominated field and satellite galaxies. Each distribution is normalized to have unit area. The shaded regions show the $1 \sigma$ error due to the Poisson noise on each bin count.

the estimators, in the median, when switching from the dispersiondominated to the rotation-dominated galaxies. For each sample, the two different estimators have a similar level of scatter, which is larger for the rotation-dominated galaxies. In general, the distributions are very similar in shape for the two estimators; however, the Wolf et al. (2010) estimator has a smaller median offset, 10th to 90th percentile spread, and standard deviation than the Walker et al. (2009) estimator, for both galaxy samples (although the difference in the scatter is much less significant for the rotation-dominated galaxies). The $\alpha$ distributions for the dispersion-dominated galaxies are approximately symmetric around the median, but the shape of the distributions is more complicated in the rotation-dominated case, where the peaks are above $\alpha=1$, and there are extended tails to low $\alpha$.
Overall, there is a $1 \sigma$ scatter for dispersion-dominated galaxies of 25 per cent when using the estimator of Walker et al. (2009), and 23 per cent for the estimator of Wolf et al. (2010). In the following, we shall investigate the dependence of the $\alpha$ distributions for dispersion-dominated galaxies on key quantities that characterize the properties of each galaxy and are most relevant to the assumptions on which the mass estimators are based.

\subsection{Stellar velocity dispersion anisotropy}

The anisotropy, $\beta$, of the stellar velocity dispersion is an important parameter in the Jeans analysis. An important feature of the mass estimators of interest here is that they are designed to be minimally sensitive to $\beta$. In Fig. 10(a), we show the $\alpha$ distributions 


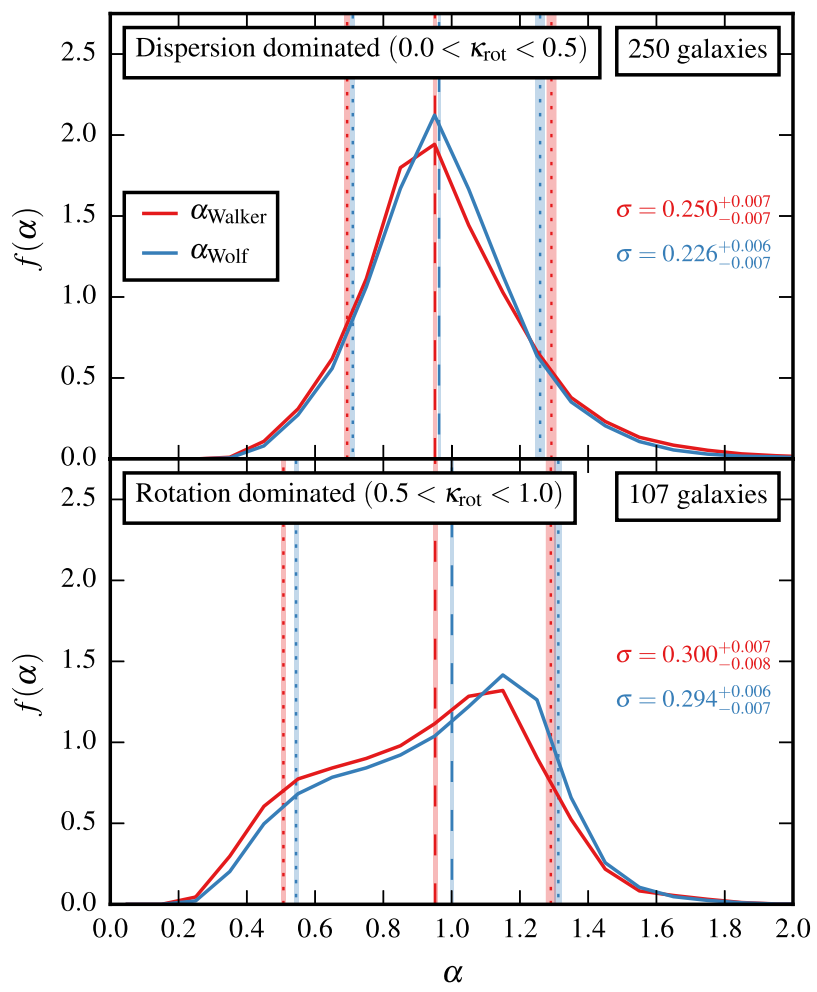

Figure 9. Distributions, $f(\alpha)$, of the estimated to true mass ratio, $\alpha$, for all galaxies, obtained by projecting over 1536 evenly distributed lines of sight. The galaxies are split into a dispersion-dominated sample (upper panel), and a rotation-dominated sample (lower panel), according to the value of $\kappa_{\text {rot }}$ (see equation 10). Different colours show the distributions obtained using the Walker et al. $\left(2009, \alpha_{\text {Walker }}\right)$ and Wolf et al. $\left(2010, \alpha_{\text {Wolf }}\right)$ estimators, as labelled. The dashed lines indicate the median $\alpha$ values for each estimator and the dotted lines indicate the 10th and 90th percentiles. The standard deviation, $\sigma$, of each distribution is given in the same colour as the lines. The shaded regions around the vertical lines and the quoted errors on $\sigma$ are the 16th-84th percentile confidence limits, derived from $10^{4}$ bootstrap samples of the galaxies for each distribution. Each projection of each galaxy contributes to the relevant distribution with equal weight, and each distribution is normalized to have unit area. The panels are labelled with the number of galaxies they include. We highlight that the $f(\alpha)$ axis limits used in Figs 10, 11, 12 and 19 vary with respect to those used here, in order to optimize the clarity of each individual figure.

for dispersion-dominated galaxies, split into bins of $\langle\beta\rangle$, which is the stellar velocity dispersion anisotropy averaged over the whole galaxy (see equation 3 ). The stellar orbits are predominantly radially biased $(\langle\beta\rangle>0)$; there are only 46 out of 250 galaxies with $\langle\beta\rangle<0$ (see Fig. 6 for the radial variation of $\beta$ in the HR simulations). For each estimator, the scatter in $\alpha$ increases with $\langle\beta\rangle$, where the difference is greatest between the intermediate and most radially biased systems, for the $\langle\beta\rangle$ intervals shown.

\subsection{Equilibrium}

In order to assess how the accuracy of the mass estimators depends on whether the galaxy of interest is in equilibrium, as assumed in the derivation of each estimator, we make use of $\tilde{\gamma}$ as defined in Section 4.4. This encodes the balance between the total stellar kinetic energy and the potential energy measured with respect to the bottom of the gravitational potential well, where galaxies with $\tilde{\gamma} \approx 1$ are assumed to be in equilibrium (the calculation of $\tilde{\gamma}$ assumes that, on average, the galaxies in our full sample are in equilibrium; see Fig. 8). The $\alpha$ distributions for dispersion-dominated galaxies divided into bins of $\tilde{\gamma}$ are shown in Fig. 10(b), where galaxies in the intermediate bin are taken to be close to equilibrium. The scatter in $\alpha$ actually increases with $\tilde{\gamma}$, such that the lowest $\tilde{\gamma}$ interval exhibits the smallest scatter for each estimator.

\subsection{Shape}

As shown in Fig. 8, the simulated galaxies span a broad range of 3D shapes. Fig. 10(c) shows the $\alpha$ distributions for dispersiondominated galaxies divided into bins of stellar sphericity, $s$. The scatter in $\alpha$ diminishes sharply as the stellar mass distribution tends towards spherical symmetry $(s=1)$. This behaviour is consistent with the expectation for the applicability of the spherical Jeans equation. Clearly, the estimators perform relatively well for galaxies that are close to spherical. However, robustly identifying such systems from projected data alone is non-trivial.

We now investigate the impact of carrying out the shape determination in projection. Adapting the procedure described in Section 4.4 for computing sphericity, we compute the reduced inertia tensor in $2 \mathrm{D}$ using the projected coordinates of each star particle on the sky; the resulting ellipse axis lengths, $a_{\mathrm{p}} \geq b_{\mathrm{p}}$, are then used to define the stellar circularity as $s_{\mathrm{p}}=b_{\mathrm{p}} / a_{\mathrm{p}}$ (a circle has $s_{\mathrm{p}}=1$ ). Fig. 10(d) shows the $\alpha$ distributions in bins of circularity, where we see a much weaker trend in the scatter as a function of shape than when the full 3D information of Fig. 10(c) is used. As such, the scatter in $\alpha$ for galaxies that appear to be very close to circular on the sky $\left(s_{\mathrm{p}}>0.9\right)$ is significantly larger than for galaxies that are actually close to spherical in $3 \mathrm{D}(s>0.75)$.

\subsection{Stellar mass}

The simple mass estimators could be expected to work equally well for stellar systems on any mass scale, provided that they satisfy the spherical Jeans equation and that more detailed assumptions relating to the stellar density distribution and the stellar velocity dispersion are reasonable (see Section 2). However, many galaxy properties scale with stellar mass, which for our simulated galaxies, albeit weakly and with a large scatter, include the level of rotational support and the 3D shape (see Figs 2 and 8). In Fig. 10(e), we show the $\alpha$ distributions for dispersion-dominated galaxies in bins of stellar mass. There is no monotonic global trend in the scatter in $\alpha$ as a function of stellar mass; however, for both estimators the scatter peaks in the intermediate mass interval.

\subsection{Structural relationship with dark matter halo}

As discussed above, Figs 9 and 10 explore the dependence of the mass estimator errors on key structural and kinematical properties of the galaxy itself, i.e. properties of the set of subhalo star particles enclosed within a sphere of radius $r_{\text {gal }}$ around the subhalo centre (including the balance between the stellar kinetic energy and the gravitational potential energy of the stars due to the mass distribution of the whole subhalo). Extending this, we now examine the dependence of the mass estimator accuracy on quantities that characterize the structural relationship between the galaxy and the dark matter component of its host subhalo.

Fig. 11(a) shows the $\alpha$ distributions for dispersion-dominated galaxies, divided into bins according to the angle, $\psi$, between the minor axis of the galaxy and that of the dark matter component of 


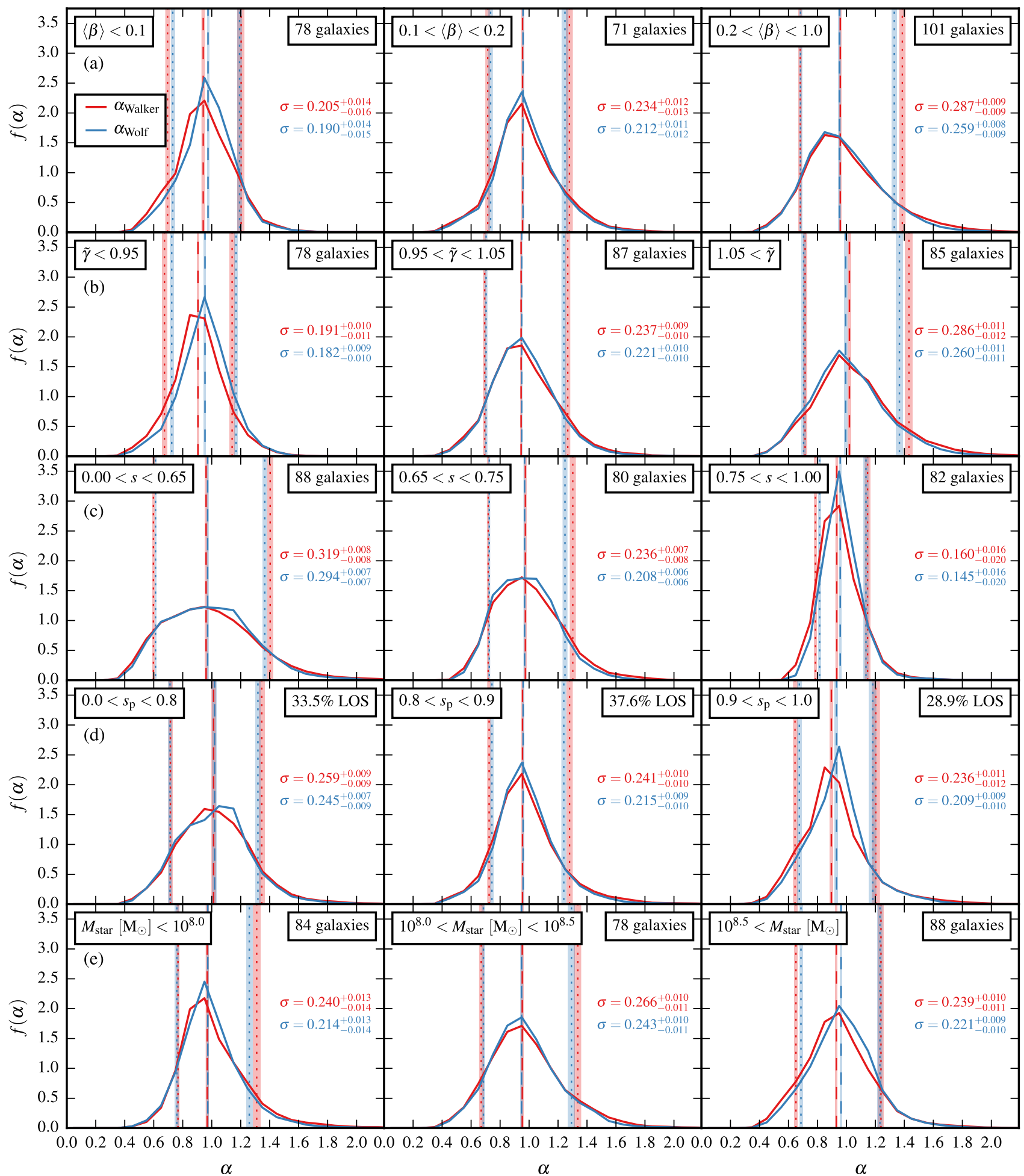

Figure 10. Distribution, $f(\alpha)$, of the estimated to true mass ratio, $\alpha$, for dispersion-dominated galaxies $\left(\kappa_{\text {rot }}<0.5\right)$, as a function of various galaxy properties. In each row, the dispersion-dominated sample is split into three bins (columns), according to (a) the mean stellar velocity dispersion anisotropy, $\langle\beta\rangle$; (b) the stellar equilibrium measure, $\tilde{\gamma}$; (c) the stellar sphericity, $s$; (d) the projected stellar circularity, $s_{\mathrm{p}}$ and (e) the stellar mass, $M_{\text {star. }}$. Each panel is labelled with the interval it considers and the number of galaxies it contains (percentage of lines of sight in the case of circularity, where a single galaxy may contribute to more than one panel). The details of the computation of the distributions and the meaning of each line are as described in the caption of Fig. 9.

its subhalo. For each mass component, the minor axis is taken to be given by the eigenvector of the reduced inertia tensor that has the smallest eigenvalue, and thus corresponds to the shortest axis length (i.e. $c$ in $s=c / a$; see Section 4.4). Note that the signs of the eigenvectors are ignored, such that the angle $\psi$ can take values from zero to $\pi / 2$. The scatter in $\alpha$ is highest for the galaxies that are close to perfect alignment with their dark matter halo $(\psi=0)$, and the scatter decreases with increasing $\psi$. 


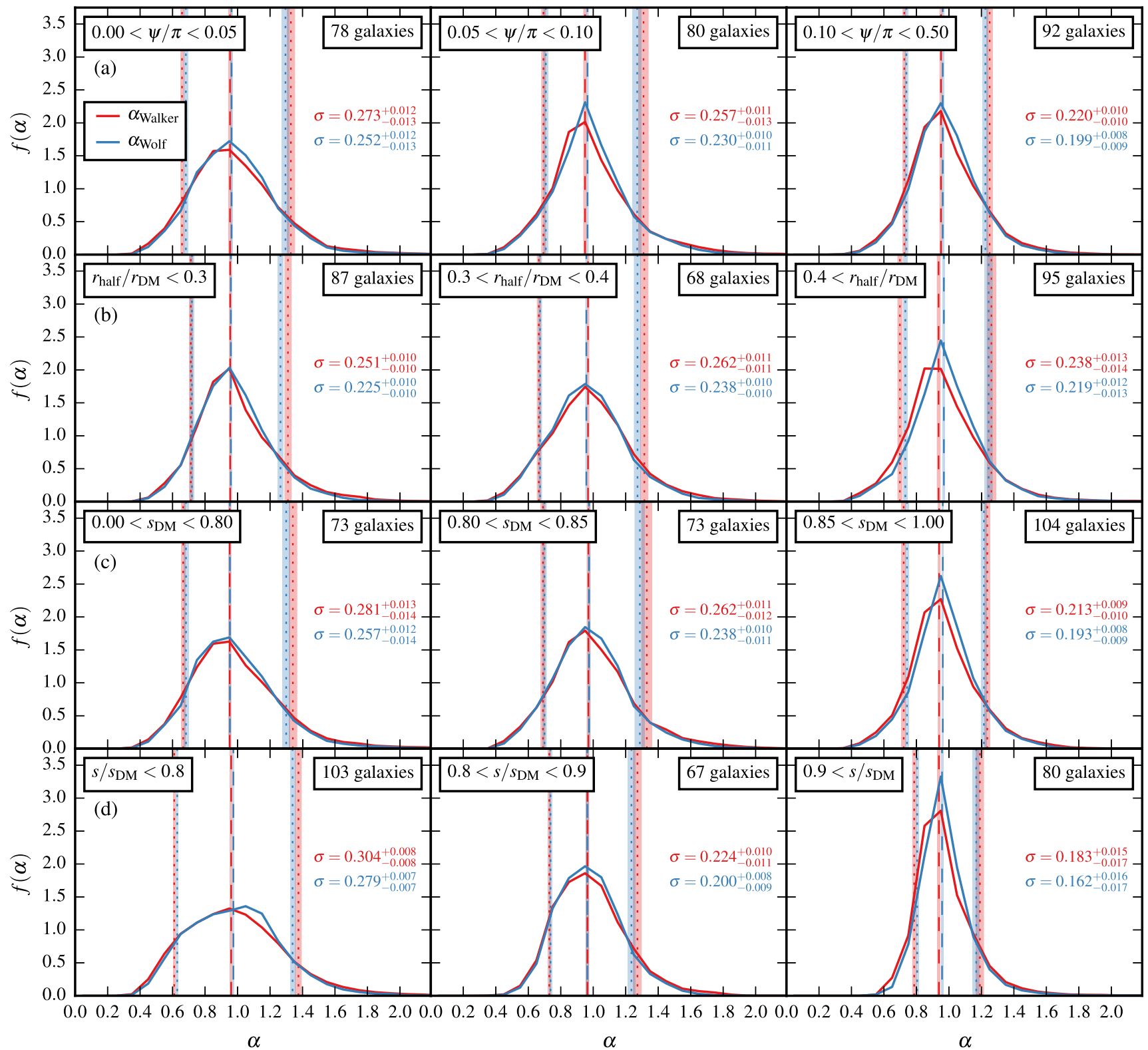

Figure 11. Distribution, $f(\alpha)$, of the estimated to true mass ratio, $\alpha$, for dispersion-dominated galaxies $\left(\kappa_{\text {rot }}<0.5\right)$, as a function of various galaxy and host subhalo dark matter properties. In each row, the dispersion-dominated galaxy sample is split into three bins (columns), according to (a) the angle between the minor axis of the galaxy and that of the dark matter mass distribution within its host subhalo, $\psi$; (b) the ratio of the stellar 3D half-mass radius, $r_{\text {half }}$, to the subhalo dark matter scale radius, $r_{\mathrm{DM}}$; (c) the subhalo dark matter sphericity, $s_{\mathrm{DM}}$ and (d) the ratio $s / s_{\mathrm{DM}}$, where $s$ is the stellar sphericity. Each panel is labelled with the interval it considers and the number of galaxies it contains. The details of the computation of the distributions and the meaning of each line are as described in the caption of Fig. 9.

In order to investigate the dependence of the estimator accuracy on how deeply the galaxy is embedded within its host subhalo, we compute a scale radius, $r_{\mathrm{DM}}$, for the subhalo dark matter mass distribution and compare this to the stellar 3D half-mass radius, $r_{\text {half }}$. Given the radius at which the circular velocity curve peaks, considering the enclosed mass in subhalo dark matter particles only, $r_{\mathrm{DM}}$ is computed as the corresponding scale radius for an exact NFW profile (Navarro, Frenk \& White 1996). ${ }^{9}$ Fig. 11(b) shows the $\alpha$

${ }^{9}$ Given the enclosed dark matter mass profile, $M_{\mathrm{DM}}(<r)$, if the radius at which the dark matter only circular velocity curve, $\sqrt{G M_{\mathrm{DM}}(<r) / r}$, peaks distributions for dispersion-dominated galaxies in bins of $r_{\text {half }} / r_{\mathrm{DM}}$, which quantifies the 'concentration' of the galaxy with respect to the dark matter distribution. The scatter in $\alpha$ peaks in the intermediate bin shown, reducing somewhat in the most concentrated bin (lowest $r_{\text {half }} / r_{\mathrm{DM}}$ ), followed by the least concentrated systems, but the differences are small.

Finally, it is interesting to examine the dependence of the estimator error on the $3 \mathrm{D}$ shape of the host dark matter halo, since the is $r_{\max (\mathrm{DM})}$, then the scale radius for an exact NFW halo with the same value of $r_{\max (\mathrm{DM})}$ is $r_{\mathrm{DM}} \simeq r_{\max (\mathrm{DM})} / 2.163$. 


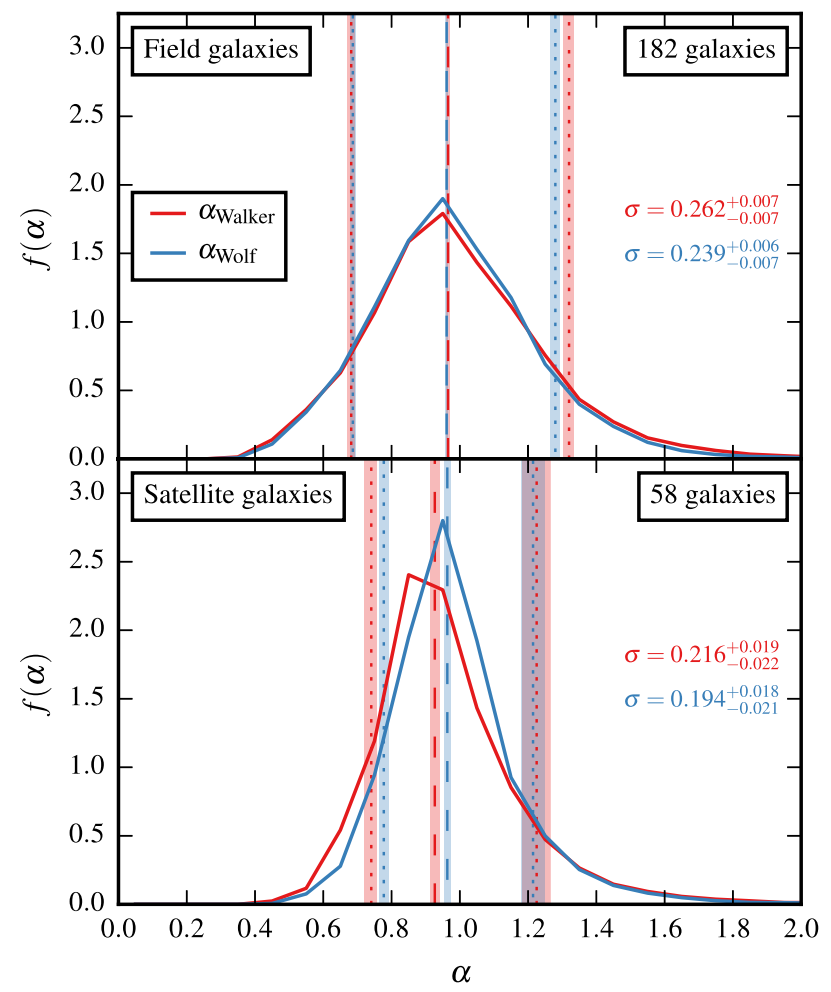

Figure 12. Distributions, $f(\alpha)$, of the estimated to true mass ratio, $\alpha$, for field (upper panel) and satellite (within $300 \mathrm{kpc}$ of MW or M31; lower panel $)$ galaxies in the dispersion-dominated sample $\left(\kappa_{\text {rot }}<0.5\right)$. The details of the computation of the distributions and the meaning of each line are as described in the caption of Fig. 9.

mass estimators assume that the dynamical system is spherically symmetric as a whole, and the scatter in $\alpha$ is found to be strongly dependent on the 3D shape of the stellar distribution (see Fig. 10c). We compute the sphericity of the subhalo dark matter distribution, $s_{\mathrm{DM}}$, in the same way as described in Section 4.4 for the stellar sphericity, s. Fig. 11(c) shows the $\alpha$ distributions for dispersiondominated galaxies in bins of $s_{\mathrm{DM}}$. The scatter in $\alpha$ decreases with increasing $s_{\mathrm{DM}}$; however, the strength of this dependence is smaller than that for the stellar sphericity, comparing to Fig. 10(c). This indicates that the shape of the stellar mass distribution has the largest impact on the estimator error, with a weaker influence from the shape of the mass distribution of the dark matter. We note that although $s$ does exhibit a weak increase on average as a function of $s_{\mathrm{DM}}$, there is a very large scatter in $s$ at a given $s_{\mathrm{DM}}$, i.e. the stellar and dark matter 3D shapes are only loosely connected. The dark matter distribution is usually closer than the galaxy to being spherically symmetric (only 24 out of 250 galaxies have $s>s_{\mathrm{DM}}$ ). Fig. 11(d) shows the $\alpha$ distributions for dispersion-dominated galaxies in bins of $s / s_{\mathrm{DM}}$. The decrease in the scatter in $\alpha$ is more significant as a function of this ratio than as a function of $s_{\mathrm{DM}}$ (cf. Fig. 11c), but less pronounced than as a function of $s$ alone (cf. Fig. 10c). Therefore, the difference between the 3D shapes of the galaxy and its host dark matter halo (quantified here by $s / s_{\mathrm{DM}}$ ) plays a role in the accuracy of simple mass estimators.

\subsection{Field versus satellite galaxies}

Fig. 12 shows the $\alpha$ distributions separately for dispersiondominated field and satellite galaxies (satellites are within $300 \mathrm{kpc}$ of a MW or M31 analogue). Although there are fewer satellites, and hence poorer statistics, the scatter in $\alpha$ is smaller for the satellite galaxies than for the field galaxies, for both estimators. This may be closely related to the fact that, on average, the satellite galaxies are closer to spherical symmetry than those in the field (see Fig. 8). There are hints that the satellites also tend to be more strongly supported by dispersion (see Fig. 2) and tend to have stellar velocity dispersions that are closer to isotropic (see Fig. 6 for HR), compared with field galaxies. We also find that the satellite galaxies tend to be more extended relative to the dark matter distribution of their subhalo (higher $r_{\text {half }} / r_{\text {DM }}$ ) than the field galaxies.

\subsection{Angular dependence}

The analysis presented so far has considered the estimator error distributions resulting from summing over all galaxies (or lines of sight) satisfying certain criteria based on integrated stellar and dark matter properties (or per-projection observable properties). We now investigate how the estimator accuracy varies with viewing angle relative to the orientation of the galaxy. Given Cartesian coordinates $(x, y, z)$, we adopt the standard convention for the spherical polar coordinates $(r, \theta, \phi)$, such that $x=r \sin (\theta) \cos (\phi), y=r \sin (\theta) \sin (\phi)$ and $z=r \cos (\theta)$. For each galaxy, we rotate the simulation coordinate system to align with the eigenvectors of the stellar reduced inertia tensor (see Section 4.4). The $z$-axis $(\theta=0)$ is aligned with the shortest principal axis (minor axis), the $x$-axis $(\theta=\pi / 2, \phi=0)$ with the longest principal axis (major axis), and the remaining principal axis with the $y$ direction $(\theta=\pi / 2, \phi=\pi / 2)$.

The upper panels of Fig. 13 show the variation in the $\alpha$ values for each estimator, relative to the mean $\alpha$ value from averaging over all lines of sight for a given galaxy, as a function of $\theta$ and $\phi$, defined using the stellar-reduced inertia tensor. The angular variations of the projected half-mass radius, $R_{\mathrm{e}}$, and the line-of-sight integrated stellar velocity dispersion, $\left\langle\sigma_{\text {los }}\right\rangle$, are also shown, again relative to the corresponding galactic means. The lower panels in Fig. 13 repeat the same analysis, but using instead the $\theta$ and $\phi$ coordinates with respect to the eigenbasis of the reduced inertia tensor of the dark matter particles within the subhalo. Thus, for a given galaxy, the $(\theta, \phi)$ coordinates contributing to the upper and lower panels of Fig. 13 differ unless the stellar and dark matter reduced inertia tensor eigenvectors are exactly aligned, with the same eigenvalue ordering (since this sets the ordering of the principal axis lengths, and hence the definitions of $\theta$ and $\phi$ ).

Looking at the upper panels of Fig. 13, it is clear that $\alpha$ is strongly sensitive to $\theta$, i.e. the angle with respect to the stellar minor axis $(z)$, and has a weaker dependence on $\phi$, i.e. the angle with respect to the major axis ( $x$; in the $x-y$ plane). The results for the two different estimators are very similar, although the estimator of Wolf et al. (2010) exhibits a slightly smaller scatter at all angles. As a function of $\theta$ (upper left panel), the mass estimates are maximally biased low (in the median for all galaxies), relative to the galactic mean values, for lines of sight coincident with the stellar minor axis $(\theta \sim 0$ and $\theta \sim \pi)$. The median estimates then smoothly increase towards the $x-y$ plane, peaking for $\theta / \pi \sim 0.5$. The scatter in $\alpha$, relative to the galactic mean, is relatively large for lines of sight along the minor axis or within the $x-y$ plane, but is minimized for $\theta / \pi \sim 0.3$ and $\theta / \pi \sim 0.7$, being approximately symmetrical around $\theta / \pi=0.5$. The scatter in both $R_{\mathrm{e}}$ and $\left\langle\sigma_{\text {los }}\right\rangle$ exhibits the same behaviour as a function of $\theta$ as the scatter in $\alpha$, with extremes at approximately the same values of $\theta$. The velocity dispersion varies with $\theta$ in the same way as $\alpha$ does, peaking for $\theta / \pi \sim 0.5$, with minima coinciding with the minor axis. However, the dependence of $R_{\mathrm{e}}$ on $\theta$ is reversed, compared to that of $\alpha$ and $\left\langle\sigma_{\operatorname{los}}\right\rangle$, such that the $R_{\mathrm{e}}$ 


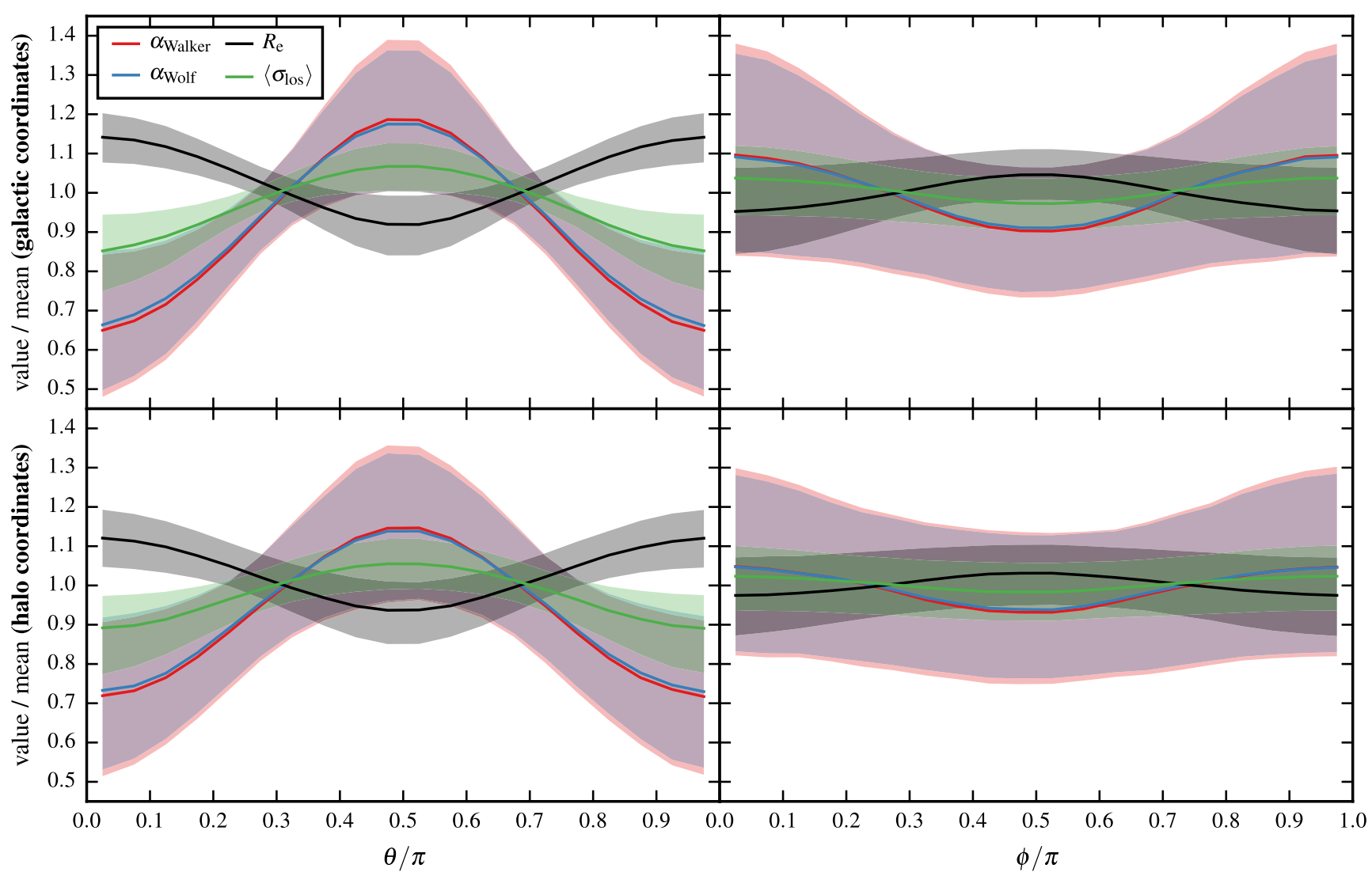

Figure 13. Angular variation of the estimated to true mass ratio for the Walker et al. $\left(2009, \alpha_{\text {Walker }}\right)$ and Wolf et al. $\left(2010, \alpha_{\text {Wolf }}\right)$ estimators, the projected stellar half-mass radius, $R_{\mathrm{e}}$, and the line-of-sight stellar velocity dispersion, $\left\langle\sigma_{\text {los }}\right\rangle$, for dispersion-dominated galaxies $\left(\kappa_{\text {rot }}<0.5\right)$, computed by projecting over 1536 evenly distributed lines of sight. The curves are shown as functions of the spherical polar angles, $\theta$ and $\phi$, where the coordinate system is aligned with the eigenbasis of the reduced inertia tensor of either the galactic star particles (upper panels) or the dark matter particles of the host subhalo (lower panels). The $z$-axis $(\theta=0)$ is aligned with the shortest principal axis, and the $x$-axis $(\theta=\pi / 2, \phi=0)$ is aligned with the longest principal axis. The spherical polar angular ranges are defined as $0 \leq \theta \leq \pi$ and $0 \leq \phi<2 \pi$. Since projections in opposite directions are equivalent in this paper, the 1536 unique lines of sight are identified by their angular coordinates in the half-sphere defined by $0 \leq \theta<\pi$ and $0 \leq \phi<\pi$ within this figure. For each galaxy, the values of $\alpha$ Walker, $\alpha_{\text {Wolf }}, R_{\mathrm{e}}$ and $\left\langle\sigma_{\mathrm{los}}\right\rangle$ for each projection have been divided by the corresponding mean value over all projections of that galaxy, before computing the curves shown. The solid lines show the median values, and the shaded regions of the same colour indicate the 16th-84th percentile spread (the $\theta$ and $\phi$ axes use the same fixed bin width, in units of $\pi$ ). Each panel makes use of the full set of 1536 lines of sight, so at fixed $\theta$ (left) or $\phi$ (right), the median and percentile range values shown result from the data for all lines of sight (with that particular $\theta$ or $\phi$ ) for all galaxies.

curve is approximately the reflection of the $\left\langle\sigma_{\text {los }}\right\rangle$ curve around the galactic mean. The coupled variation of $R_{\mathrm{e}}$ and $\left\langle\sigma_{\text {los }}\right\rangle$ suppresses the variation in the product $R_{\mathrm{e}}\left\langle\sigma_{\mathrm{los}}\right\rangle^{2}$, and hence the variation in the estimated mass (see equations 13 and 14). The $\alpha, R_{\mathrm{e}}$ and $\left\langle\sigma_{\text {los }}\right\rangle$ curves intersect (with values $\sim 1$ ) for $\theta$ close to where the scatter in each quantity is minimized $(\theta / \pi \sim 0.3$ and $\theta / \pi \sim 0.7)$.

The results as a function of $\phi$ in galactic coordinates (upper right panel in Fig. 13) are similar to those just described for the $\theta$ dependence, but with smaller median offsets from unity and larger scatters, exhibiting extremes such that $\alpha$ and $\left\langle\sigma_{\text {los }}\right\rangle$ are maximized for $\phi \sim 0$ and $\phi \sim \pi$ (i.e. for lines of sight in the $x-z$ plane), and minimized for $\phi / \pi \sim 0.5$ (i.e. the $y-z$ plane); while the $\phi$ values of the turning points are shifted by an angle of $\pi / 2$ relative to this for $R_{\mathrm{e}}$. Note that since projections in opposite directions are equivalent for our purposes, the angular coordinates of the 1536 unique lines of sight are evaluated within the half-sphere defined by $0 \leq \theta<\pi$ and $0 \leq \phi<\pi$ in Fig. 13 .

Using instead the coordinate system based on the subhalo dark matter, as shown in the lower panels of Fig. 13, results in dependences of $\alpha, R_{\mathrm{e}}$ and $\left\langle\sigma_{\text {los }}\right\rangle$ on the spherical polar angles that are qualitatively very similar to the data shown in the upper panels, where the coordinates based on the galactic star particles are used. However, the strengths of the trends seen in galactic coordinates are diluted when switching to the halo-based coordinates, such that the median variations are reduced for each quantity shown (i.e. the solid lines are slightly closer to unity). The scatter around the median also increases for each quantity at all angles when switching to the halo coordinates, except for $\phi / \pi \lesssim 0.2$ and $\phi / \pi \gtrsim 0.8$, where for each curve shown, the 16 th-84th percentile width actually decreases. The differences between the upper and lower panels in Fig. 13 indicate that the accuracy of the mass estimators, for a given line of sight, is more sensitive to the shape and relative alignment of the galaxy, rather than the corresponding properties of the dark matter (sub)halo in which the galaxy is embedded.

Fig. 14 shows the mean $\alpha$ value for the Walker et al. (2009) estimator, as a function of $\cos (\theta)$ and $\phi$, for all dispersion-dominated galaxies, using the coordinate system based on the reduced inertia tensor of the stars within each galaxy, as in the upper panels of Fig. 13 (but note that Fig. 13 considers the $\alpha$ values relative to the galactic mean, for each galaxy, while in Fig. 14 the actual individual $\alpha$ values are used directly to compute the mean for each pixel). Since the lines of sight are drawn from an evenly spaced spherical 


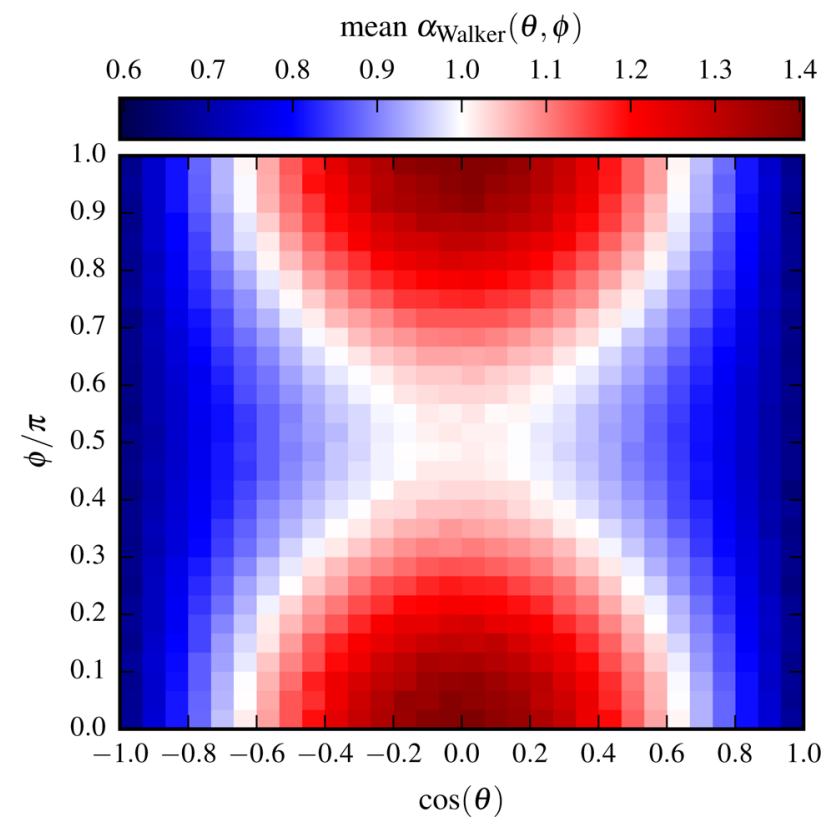

Figure 14. Estimated to true mass ratio, $\alpha_{\text {Walker }}(\theta, \phi)$, resulting from applying the estimator of Walker et al. (2009) to all galaxies in our dispersiondominated sample $\left(\kappa_{\text {rot }}<0.5\right)$, by projecting over 1536 evenly distributed lines of sight for each galaxy. $\theta$ and $\phi$ are the spherical polar angles in the galactic coordinate system defined by the eigenbasis of the stellar-reduced inertia tensor, where the $z$-axis $(\theta=0)$ is aligned with the shortest stellar principal axis, and the $x$-axis $(\theta=\pi / 2, \phi=0)$ is aligned with the longest principal axis. The angular ranges are defined as $0 \leq \theta \leq \pi$ and $0 \leq \phi<2 \pi$; however, since projections in opposite directions are equivalent in the context of our analysis, we consider the coordinates of the set of 1536 unique lines of sight within the half-sphere defined by $0 \leq \theta<\pi$ and $0 \leq \phi<\pi$ in the grid shown here (extending the grid to $\phi=2 \pi$ would result in repetition of the data). Since the lines of sight result from an evenly distributed spherical tessellation, plotting $\phi$ versus $\cos (\theta)=z / r$ ensures that the number of projections per grid pixel is approximately constant. The $\alpha_{\text {Walker }}(\theta, \phi)$ value shown in each pixel is the mean over all projections of each galaxy within the relevant angular range. The colour scale is centred on a mass ratio of unity (i.e. white for accurate mean estimates, blue for mean underestimates and red for mean overestimates). The results for the Wolf et al. (2010) estimator are very similar to those shown here. The distributions of the mass ratios, $\alpha$, for both estimators are shown in the upper panel of Fig. 9 (summing over all projections), and the $\alpha$ variations relative to the galactic mean are shown in the upper panels of Fig. 13 (integrating over $\theta$ and $\phi$ separately).

tessellation, they must evenly sample the $\cos (\theta)$ versus $\phi$ plane, and so the pixels in Fig. 14 each consider approximately the same number of data points (galaxy projections). The results for the Wolf et al. (2010) estimator are very similar to those shown in Fig. 14. As can be seen in Fig. 14, the most accurate mean mass estimates form an hourglass shape in the $\cos (\theta)-\phi$ plane (white). The masses are most heavily underestimated for $\cos (\theta) \sim \pm 1$ (blue; corresponding to lines of sight along the stellar minor axis), and most heavily overestimated for $\cos (\theta) \sim 0$ with $\phi / \pi \sim 0$ or $\phi / \pi \sim 1$ (red; corresponding to lines of sight along the stellar major axis). Lines of sight coincident with the stellar principal axis of intermediate length, where $\cos (\theta)=0$ and $\phi / \pi=0.5$ (or $\phi / \pi=1.5$; not shown), correspond to the centre of the grid shown in Fig. 14, where the mean mass estimates are relatively close to perfect accuracy.

Fig. 15 is the same as Fig. 14, except that it shows the standard deviation of the $\alpha$ values, as a function of $\cos (\theta)$ and $\phi$; otherwise the calculations involved are identical for the two figures. It can be seen in Fig. 15 that the scatter in $\alpha$ is highest for lines of sight

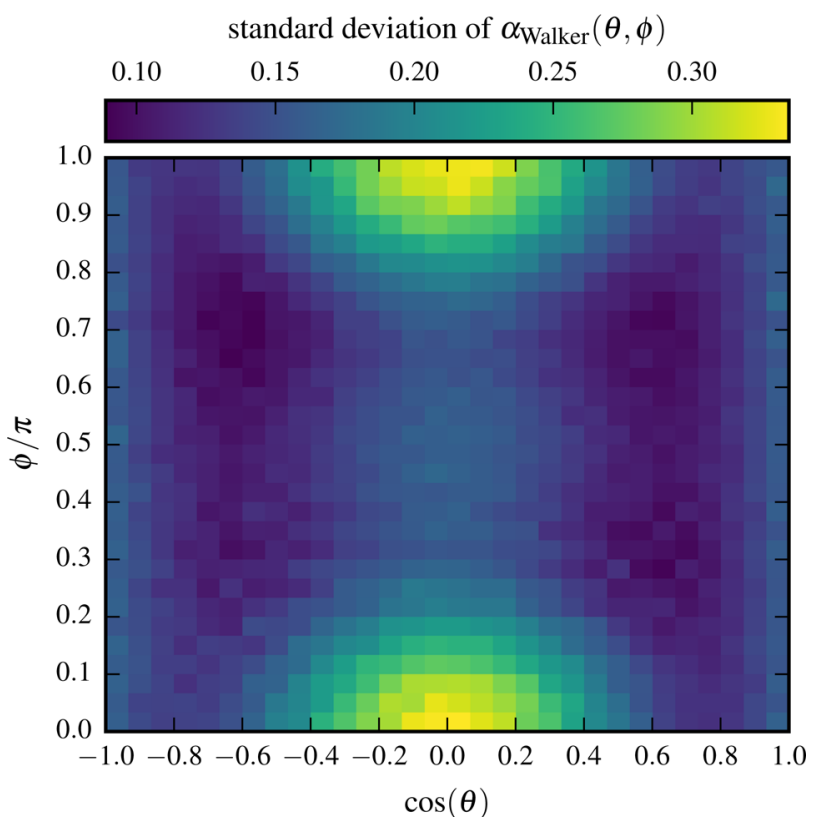

Figure 15. The same as Fig. 14, but showing instead the standard deviation of the $\alpha_{\text {Walker }}(\theta, \phi)$ values in each pixel, rather than the mean value. The details of the analysis are otherwise exactly as described in Fig. 14.

along the stellar major axis, and lowest for the two regions with $\cos (\theta) \sim \pm 0.6$ (i.e. $\theta / \pi \sim 0.3$ and $\theta / \pi \sim 0.7$ ) and $0.2 \lesssim \phi / \pi \lesssim 0.8$ (in keeping with the upper panels of Fig. 13). The scatter for lines of sight coincident with the stellar minor axis and the intermediate stellar principal axis is fairly similar, in stark contrast to the major axis. Comparing Figs 14 and 15, we see that the locations of the largest scatter in $\alpha$ are also the locations of the highest mean $\alpha$ values. For the three stellar principal axes, the dynamical masses are either overestimated with a large scatter (major axis), underestimated with a modest scatter (minor axis) or in fact close to accurate with a modest scatter (intermediate axis).

\subsection{Summary and discussion}

To summarize the preceding results, Fig. 16 shows $\alpha$ for each estimator as a function of various galaxy properties, in bins of approximately equal numbers of galaxies (or sightlines). The first panel shows the dependence on $\kappa_{\text {rot }}$ for the full galaxy sample, and the subsequent panels consider only the dispersion-dominated galaxies $\left(\kappa_{\text {rot }}<0.5\right)$. The distinction between the two estimators is small, although where they deviate, the Wolf et al. (2010) estimator has the smallest bias and scatter in almost all cases.

Considering the scatter, the estimators are most precise for dispersion-supported galaxies that are close to spherical, have at most mildly radially biased stellar velocity dispersions $(\langle\beta\rangle \lesssim 0.2)$, and have relatively low stellar kinetic energy for their gravitational potential energy. The strongest dependence of the scatter is on the 3D shape of the stellar mass distribution, of which almost all useful information is lost in projection. Considering only the dispersiondominated galaxies, as shown in Fig. 16, the scatter also reduces for galaxies that are misaligned with respect to their host dark matter halo, and for galaxies that are highly extended, in units of their halo scale radius. It is interesting to note that the (dispersion-dominated) satellite galaxies exhibit a lower $\alpha$ scatter overall compared to their counterparts in the field (see Fig. 12). Satellite galaxies also tend to be closer to spherical and tend to be more extended relative to their 

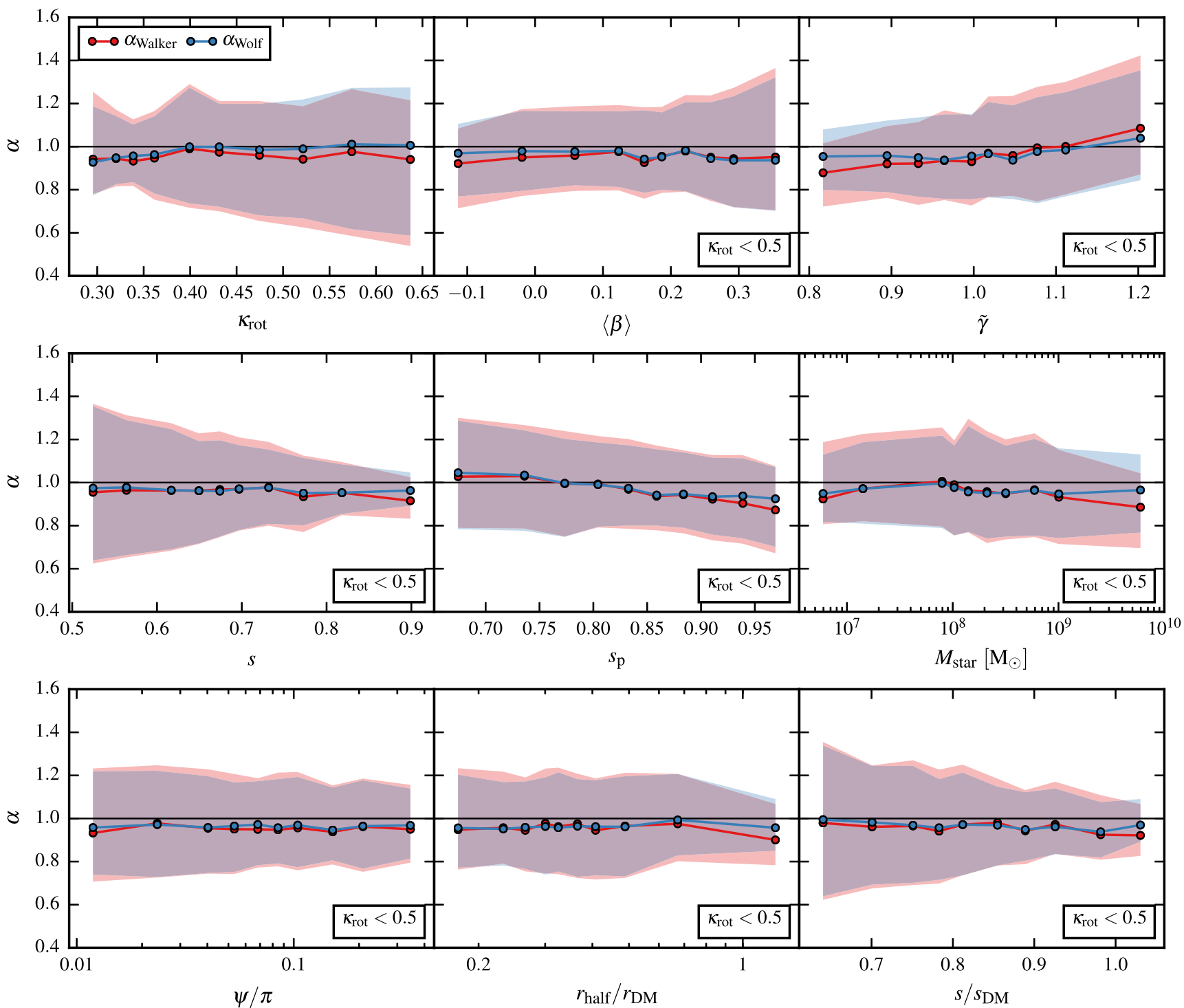

Figure 16. Ratio, $\alpha$, of the estimated to true mass obtained using the estimators of Walker et al. (2009, $\left.\alpha_{\text {Walker }}\right)$ and Wolf et al. (2010, $\left.\alpha_{\text {Wolf }}\right)$, by projecting over 1536 evenly distributed lines of sight, as a function of various galaxy and subhalo dark matter properties. The properties shown are the stellar kinematic measure used to discriminate between galaxies with different levels of rotational support $\left(\kappa_{\text {rot }}\right)$, the mean stellar velocity dispersion anisotropy $(\langle\beta\rangle)$, the stellar equilibrium measure $(\tilde{\gamma})$, the stellar sphericity $(s)$, the projected stellar circularity $\left(s_{\mathrm{p}}\right)$, the stellar mass $\left(M_{\mathrm{star}}\right)$, the angle between the stellar and subhalo dark matter minor axes $(\psi)$, the ratio of the stellar and dark matter scale radii $\left(r_{\text {half }} / r_{\mathrm{DM}}\right)$, and the ratio of the stellar and dark matter sphericities $\left(s / s_{\mathrm{DM}}\right)$. The first panel shows $\alpha$ versus $\kappa_{\text {rot }}$ for all galaxies; all other panels consider only those galaxies whose stellar motions are dominated by dispersion $\left(\kappa_{\text {rot }}<0.5\right)$. For each estimator (colours), the solid lines show the median mass ratios and the shaded regions the 16th-84th percentile spread. The bin edges are evenly spaced percentiles of the quantity on the horizontal axis, and the bin centres (points) are the median values in each bin.

host dark matter halo, compared to the field galaxies (the highest $r_{\text {half }} / r_{\text {DM }}$ bin in Fig. 16 contains 21 satellite galaxies and only 4 field galaxies). We note that the main physical galaxy properties of interest are related at some level: galaxies that are close to spherical tend to have velocity dispersions that are close to isotropic and have relatively little rotational support. The scatter in $\alpha$ is much more sensitive to the shape of the galaxy than to that of the mass distribution of the dark matter within its host subhalo, such that there remain strong trends in the scatter in $\alpha$ as a function of the ratio of stellar and dark matter sphericities, $s / s_{\mathrm{DM}}$.

Any trends in the median $\alpha$ values shown in Fig. 16 are weak. However, considering the dependence on the projected stellar circularity, $s_{\mathrm{p}}$, the bias towards overestimation of the mass for galaxies that are very elongated on the sky gradually decreases with increas- ing circularity (we note that this trend is exaggerated if we instead consider the full galaxy sample, including the rotation-dominated galaxies). The median $\alpha$ also exhibits an increase with increasing $\tilde{\gamma}$ (i.e. for increasing stellar kinetic energy relative to the stellar gravitational potential energy, at fixed stellar mass).

As shown in Fig. 13 (upper panels), the value of $\alpha$ is strongly sensitive to the viewing angle, $\theta$, relative to the stellar minor axis, $z$, such that the estimated masses are lowest for lines of sight close to the minor axis, and highest for lines of sight in the $x-y$ plane. The dependence of $\alpha$ on the other spherical polar viewing angle, $\phi$, relative to the stellar major axis, $x$ (in the $x-y$ plane), is much weaker than the dependence on $\theta$, but shows similar behaviour. As shown in Figs 14 and 15, the dynamical masses are overestimated with a large scatter for lines of sight coincident with the major axis, 
underestimated with a modest scatter for the minor axis, and close to accurate with a modest scatter for the intermediate principal axis.

We note that Wolf et al. (2010) suggest an error of around 0.05 dex (12 per cent; cf. Fig. 16) for their estimator (in addition to the uncertainty due to the errors on the measured size and velocity dispersion), in the special case where the unknown $\beta(r)$ has an extremum within the stellar distribution, or $\sigma_{\text {los }}(R)$ has not been measured over the full extent of the galaxy (i.e. the dispersion much beyond $R \sim R_{\mathrm{e}}$ is not included when $\left\langle\sigma_{\text {los }}\right\rangle$ is computed). Otherwise, Wolf et al. (2010) advocate considering only the measurement errors on the velocity dispersion and half-light radius when evaluating the uncertainties in masses obtained using their estimator.

Kowalczyk et al. (2013) apply a modified version of the estimator of Wolf et al. (2010) to idealized simulations of dSphs in a static MW potential, and find that the error decreases as the shape of the stellar mass distribution tends towards spherical symmetry, as also found here. However, they also find that for the simulated dSphs there is no clear dependence of the error on the level of stellar rotation or velocity dispersion anisotropy, while we do detect such dependences here (see Fig. 16).

Laporte et al. (2013a) investigate the accuracy of the Walker et al. (2009) estimator for idealized stellar distributions placed in dark matter subhaloes from the Aquarius simulations, finding fluctuations of between 10 and 20 per cent with respect to the true mass. An interesting result of this study, which naturally takes into account the triaxiality of dark matter haloes formed in cosmological simulations, is that it finds that the enclosed dynamical mass is more strongly overestimated for stellar populations that are more deeply embedded (more highly concentrated) within their dark matter halo, concurring with the spherically symmetric tests of Walker \& Peñarrubia (2011). However, we do not detect significant evidence of such a trend in the APOSTLE simulations (see the $r_{\text {half }} / r_{\text {DM }}$ panel of Fig. 16). Additionally, Laporte et al. (2013a) demonstrate variations of the estimated mass, $R_{\mathrm{e}}$, and $\left\langle\sigma_{\text {los }}\right\rangle$, as a function of viewing angle, that are in good qualitative agreement with the results presented in Section 5.8.

Lyskova et al. (2015) apply the Wolf et al. (2010) estimator to cosmological zoom simulations of 40 isolated elliptical galaxies, finding a $1 \sigma$ scatter of around 8 per cent for $V_{\mathrm{c}}\left(4 R_{\mathrm{e}} / 3\right)$ (note that the fractional error on $V_{\mathrm{c}}(r)$ is half that on $M(<r)$, assuming zero error on the radius), with a bias of 3 per cent above the true circular velocity (cf. Fig. 16).

Our results complement these earlier studies, but we have used a large sample of realistic galaxies with a broad range of stellar masses, formed in a self-consistent $\Lambda \mathrm{CDM}$ cosmological context, in the environment of the Local Group, using sophisticated treatments of the baryonic physics important for galaxy formation.

\section{EFFECTS OF UNCERTAINTIES}

We now explore the impact of the systematic uncertainties associated with the simple mass estimators on key results in the literature that are based on applying the estimators to observational data. Despite the high resolution of the APOSTLE simulations, our sample of galaxies does not include systems with stellar masses smaller than that of Sculptor (due to our chosen resolution threshold; see Section 3.3 and Fig. 7). However, the estimator error has no clear dependence on stellar mass (see Fig. 10e). Therefore, we assume that the uncertainties explored in Section 5 remain relevant for galaxies that are fainter than those in our sample.
Table 2. Dynamical mass, $M_{\text {Wolf }}$, within the $3 \mathrm{D}$ half-light radius $\left(r_{\text {half }} \approx\right.$ $4 R_{\mathrm{e}} / 3$ ) of dSph satellites of the MW, according to the estimator of Wolf et al. (2010), as given in equation (9). The estimates have been computed using the projected half-light radii, $R_{\mathrm{e}}$ (as reproduced here), and line-of-sight velocity dispersions tabulated by Wolf et al. (2010). $\sigma_{M}$ is the $1 \sigma$ fractional error on $M_{\text {Wolf }}$, obtained by propagating the observational uncertainties on the size and dispersion measurements through equation (9), approximating the errors as symmetric. To illustrate the impact of the systematic errors explored in this paper, the final column gives the fractional error, $\tilde{\sigma}_{M}=\left(\sigma_{M}^{2}+\sigma_{\text {sys }}^{2}\right)^{1 / 2}$, on the estimated mass if a representative systematic error of $\sigma_{\text {sys }}=0.2$ is assumed for the estimated to true mass ratio (ignoring any bias; see Fig. 12). The galaxies are listed in the order of decreasing $M_{\text {Wolf }}$.

\begin{tabular}{lcccc}
\hline Galaxy & $R_{\mathrm{e}}(\mathrm{pc})$ & $\log _{10}\left(M_{\text {Wolf }}\left[\mathrm{M}_{\odot}\right]\right)$ & $\sigma_{M}$ & $\tilde{\sigma}_{M}$ \\
\hline Fornax & $714_{-40}^{+40}$ & 7.88 & 0.07 & 0.21 \\
Ursa Minor & $445_{-44}^{+44}$ & 7.74 & 0.14 & 0.25 \\
Sextans & $768_{-47}^{+47}$ & 7.56 & 0.10 & 0.23 \\
Canes Venatici I & $564_{-36}^{+36}$ & 7.48 & 0.15 & 0.25 \\
Leo I & $295_{-49}^{+49}$ & 7.35 & 0.19 & 0.27 \\
Sculptor & $282_{-41}^{+41}$ & 7.33 & 0.15 & 0.25 \\
Draco & $220_{-11}^{+11}$ & 7.32 & 0.11 & 0.23 \\
Bootes I & $242_{-20}^{+22}$ & 7.26 & 0.50 & 0.54 \\
Ursa Major I & $318_{-39}^{+50}$ & 7.23 & 0.30 & 0.36 \\
Carina & $254_{-28}^{+28}$ & 6.99 & 0.13 & 0.24 \\
Leo II & $177_{-13}^{+13}$ & 6.86 & 0.17 & 0.26 \\
Leo T & $115_{-17}^{+17}$ & 6.81 & 0.44 & 0.48 \\
Ursa Major II & $140_{-25}^{+25}$ & 6.77 & 0.45 & 0.50 \\
Hercules & $229_{-19}^{+19}$ & 6.74 & 0.36 & 0.41 \\
Coma Berenices & $77_{-10}^{+10}$ & 6.18 & 0.37 & 0.42 \\
Canes Venatici II & $74_{-10}^{+14}$ & 6.16 & 0.46 & 0.51 \\
Leo IV & $116_{-34}^{+26}$ & 6.07 & 1.06 & 1.08 \\
Segue 1 & $29_{-5}^{+8}$ & 5.70 & 0.56 & 0.59 \\
Willman 1 & $25_{-6}^{+5}$ & 5.57 & 0.50 & 0.54 \\
\hline
\end{tabular}

\subsection{Dynamical masses of MW dSphs}

Wolf et al. (2010) find that simply propagating the observational errors on the half-light radius and the line-of-sight velocity dispersion through their estimator equation yields a similar uncertainty for the estimated dynamical mass to that obtained from their full Jeans analysis (which, like the estimator, assumes spherical symmetry). The authors argue that this is consistent with their claim that the uncertainty on the recovered mass is dominated by observational errors, rather than underlying systematic effects. However, as we have demonstrated, the estimator's precision is sensitive to various properties of the target galaxy.

To illustrate the significance of the systematic error on the estimated mass, Table 2 lists the mass within the 3D half-light radius for dSphs of the MW, which we compute using equation (9), given the projected half-light radius and line-of-sight velocity dispersion values tabulated by Wolf et al. (2010). ${ }^{10}$ For each galaxy, we list the $1 \sigma$ fractional error, $\sigma_{M}$, on the estimated mass, obtained by propagating the observational errors through equation (9), as advocated by Wolf et al. (2010). The final column gives the fractional error on the mass if, in addition, we add in quadrature a representative $1 \sigma$ systematic error of 20 per cent in the estimated to true mass

\footnotetext{
${ }^{10}$ Note that Wolf et al. (2010) tabulate the mass within the 3D half-light radius from their full Jeans analysis, rather than from their estimator.
} 
ratio. This fiducial value for the scatter is appropriate on average for the dispersion-dominated satellite galaxies in the simulations (see Fig. 12), or all dispersion-dominated galaxies that appear nearcircular on the sky (see Fig. 10d). For many of the bright satellites, the observational errors on the measured quantities are sufficiently small that the increase in the overall mass error due to including the representative systematic uncertainty is similar in size to the original mass error. However, in many other cases the original error is so large that the contribution from the systematic uncertainty is relatively insignificant.

\subsection{Density profiles of Sculptor and Fornax}

Walker \& Peñarrubia (2011) employ a Markov Chain Monte Carlo (MCMC) technique to infer projected half-light radii and velocity dispersions for chemo-dynamically distinct stellar subpopulations in the Sculptor and Fornax dSphs. In each galaxy, there is a centrally concentrated, kinematically cold, metal-rich stellar component (population 1), and a more extended, metal-poor component, with higher velocity dispersion (population 2). The procedure used by Walker \& Peñarrubia (2011) treats each galaxy as a superposition of two spherically symmetric stellar populations (each described by a Plummer profile), with Gaussian velocity and metallicity distributions. Assuming that the two populations independently trace the gravitational potential, the resulting size and dispersion values define a mass slope, $\Gamma=\Delta \log M(<r) / \Delta \log r$, using equation (1), where the actual values of $\lambda$ and $\mu$ are not relevant for computing the slope:

$\Gamma=\frac{\log \left[M\left(<r_{2}\right) / M\left(<r_{1}\right)\right]}{\log \left[r_{2} / r_{1}\right]} \approx 1+\frac{\log \left[\sigma_{2}^{2} / \sigma_{1}^{2}\right]}{\log \left[r_{2} / r_{1}\right]}$.

Here, $r_{1}$ and $r_{2}$ are $3 \mathrm{D}$ radii equal to (or some multiple of) the projected half-light radii, $R_{\mathrm{e}}$, of populations 1 and 2 , and $\sigma_{1}$ and $\sigma_{2}$ are the mean velocity dispersions, $\left\langle\sigma_{\text {los }}\right\rangle$, of each population, respectively. Building a posterior probability distribution function, $P(\Gamma)$, using the set of $r_{1}, r_{2}, \sigma_{1}$ and $\sigma_{2}$ values at each point in the MCMC chains, Walker \& Peñarrubia (2011) find median slopes of $\Gamma=2.61_{-0.37}^{+0.43}$ for Fornax and $\Gamma=2.95_{-0.39}^{+0.51}$ for Sculptor, where the ranges indicate the 16th-84th percentile confidence intervals.

A measurement of $\Gamma$ using the masses enclosed at two non-zero radii places an upper limit on the inner logarithmic slope, $\gamma_{\mathrm{DM}}$, of the dark matter density profile, such that $\gamma_{\mathrm{DM}}<3-\Gamma$. Given that $\gamma_{\text {DM }}=1$ for an NFW density profile (Navarro et al. 1996), while e.g. $\gamma_{\mathrm{DM}}=0$ for a constant density core, Walker \& Peñarrubia (2011) exclude NFW (or steeper) profiles with confidence of (at least) 95.9 and 99.8 per cent for Fornax and Sculptor, respectively. These significance values, $s\left(\gamma_{\mathrm{DM}}\right)$, are computed as,

$s\left(\gamma_{\mathrm{DM}}\right)=\frac{\int_{3-\gamma_{\mathrm{DM}}}^{\infty} P(\Gamma) \mathrm{d} \Gamma}{\int_{-\infty}^{\infty} P(\Gamma) \mathrm{d} \Gamma}$.

If the estimates of the enclosed dynamical masses for the two stellar populations each happen to be biased by exactly the same (arbitrary) factor, with respect to the corresponding true masses, then clearly such a bias cancels out in the calculation of $\Gamma$, i.e. the recovered mass profile slope is insensitive to any coherent bias in the estimates of the enclosed masses at the two radii (see equation 15). Therefore, only differences in the enclosed mass estimation biases for the two populations are relevant in the context of the methodology of Walker \& Peñarrubia (2011).

Laporte et al. (2013a) generate stellar distribution functions in dark matter subhaloes drawn from the Aquarius simulations, in order to assess the sensitivity of the accuracy of the dual population method of Walker \& Peñarrubia (2011) to the triaxiality of the gravitational potential (i.e. the lack of spherical symmetry in the dark matter distribution). The lack of symmetry tends to introduce an anticorrelation between $R_{\mathrm{e}}$ and $\left\langle\sigma_{\text {los }}\right\rangle$ that suppresses the error on the recovered mass (see also Fig. 13 and Section A2). Laporte et al. (2013a) argue that the level of spherical symmetry is not important to the estimator accuracy, in the content of the analysis of Walker \& Peñarrubia (2011), despite the fact that they obtain fluctuations of between 10 and 20 per cent for the estimated masses (cf. Fig. 16). These authors find, as in the spherically symmetric tests of Walker \& Peñarrubia (2011), that $\Gamma$ tends to be systematically underestimated, and thus the exclusion confidences for NFW profiles in Sculptor and Fornax are deemed to be conservative (i.e. the results for $\Gamma$ can be considered as reliable lower limits, corresponding to reliable upper limits on the density slope, since $\gamma_{\mathrm{DM}}<3-\Gamma$ ). This is because the enclosed mass tends to be more strongly overestimated for tracer populations that are more deeply embedded. However, as noted in Section 5.9, we do not find any significant evidence of such a trend in the bias in the estimated to true mass ratio $(\alpha)$ as a function of the concentration of the stellar distribution $\left(r_{\text {half }} / r_{\mathrm{DM}}\right)$ for the dispersion-dominated galaxies within our simulations (see Fig. 16).

An important aspect of the tests of Laporte et al. (2013a), which use idealized independent stellar distributions placed in realistic dark matter haloes, is that the shapes (equidensity surfaces) of the stellar populations exactly trace the gravitational potential, and so the shapes of any two stellar populations generated in this way are inherently closely correlated, by construction (Laporte et al. 2013b). This close coupling assumed between the two populations may produce artificially small errors on the recovered mass slope, as it does not take into account the possibility that the stellar mass may be distributed in a way that does not exactly follow the contours in the potential, and that the two populations may have quite different $3 \mathrm{D}$ shapes and relative alignments.

Kowalczyk et al. (2013) carry out a similar study to that of Laporte et al. (2013a), but making use of idealized simulations of $\mathrm{dSph}$ galaxies orbiting in a static MW potential. The galaxies are initialized as perfect stellar discs, embedded in spherical dark matter subhaloes, with the two dSph stellar populations assumed to originate from the inner and the outer disc. These authors use the velocity dispersion measured within $R_{\mathrm{e}}$ when computing $\Gamma$, in contrast to the method of Walker \& Peñarrubia (2011), which uses the dispersion averaged over the whole galaxy. ${ }^{11}$ They find that $\Gamma$ can be biased both low and high with respect to the true slope, depending on the line of sight, and that the results are most accurate for galaxies that are close to spherical. If $\Gamma$ was overestimated for Sculptor or Fornax by Walker \& Peñarrubia (2011) then the confidence with which they exclude an inner NFW slope would be artificially high. However, as pointed out by Laporte et al. (2013a), since Kowalczyk et al. (2013) use the dispersion measured within $R_{\mathrm{e}}$ for each population, this may yield significantly different results to the method used by Walker $\&$ Peñarrubia (2011). Indeed, Laporte et al. (2013a) demonstrate that if they measure the dispersion within $R_{\mathrm{e}}$ then their analysis using the Aquarius simulations can also overestimate $\Gamma$. Note that in this case $\Gamma$ is more accurate (i.e. less biased), albeit typically with a larger scatter. However, since the objective of applying the methodology of Walker \& Peñarrubia (2011) is to place

\footnotetext{
${ }^{11}$ Kowalczyk et al. (2013) refer to the estimator they use as that of Wolf et al. (2010). In fact, Wolf et al. (2010) advocate averaging the dispersion over all stars belonging to the galaxy.
} 
conservative upper limits on the inner density profile slopes, in the context of ruling out NFW profiles, more accurate values of $\Gamma$ are deemed to be undesirable, since in this case the slope is much more likely to be overestimated, hence leading to unreliable (i.e. non-conservative) NFW profile exclusion confidence values.

If it can be assumed that the biases in the estimated masses for the two stellar populations are each determined entirely by the 'choice' of sightline orientation relative to the whole dynamical system (galaxy) for a single observer, and no other factors are relevant (including e.g. the 3D shapes and relative orientations of the distinct stellar populations), then the mass bias will of course be identical for the two stellar populations, ${ }^{12}$ and the line-of-sight choice will not affect the recovered $\Gamma$ value. However, it seems somewhat unlikely that this assumption would hold in general for real galaxies, since the two metallicity populations are likely to have formed by different processes and at different times (Benítez-Llambay et al. 2016). The two populations might have different levels of dispersion support, velocity dispersion anisotropy, 3D shapes, alignments relative to their host halo and to each other, and may be in different dynamical states relative to equilibrium; each factor potentially introducing scatter into the two mass estimates. Differences in these important properties between the two populations could result in the introduction of additional uncorrelated biases into the two recovered mass values, and hence lead to propagation of unanticipated errors into the measurement of $\Gamma$.

Clearly, the validity of assuming that the two populations yield mass estimates with identical (and hence irrelevant) random biases depends inversely on the extent to which it is valid to assume that the populations are independent. None the less, if we are to exclude NFW profiles within dSphs with multiple stellar populations, then surely the conservative approach is to assume that the two populations are indeed independent, with uncorrelated random mass errors. In fact, the APOSTLE galaxies studied here exhibit a range of alignment angles and 3D shapes relative to their host haloes (see Fig. 16). Therefore, it seems entirely plausible that the dual stellar populations found in certain $\mathrm{dSphs}$ could have different orientations and shapes with respect to each other (and their host halo), depending on their physical origins. We plan to return to this important issue in a subsequent paper.

In the special (and perhaps unlikely) limiting case where the two stellar populations are in perfect morphological alignment with each other, Fig. 14 corresponds to the prediction from our simulations for the mean value of the estimated to true mass ratio for the Walker et al. (2009) estimator, as a function of viewing angle relative to the (here assumed perfectly aligned) principal axes of the stellar populations. Even though this mean bias would cancel out when computing $\Gamma$, there is also a substantial scatter around the mean expectation, for any randomly chosen line of sight, as shown in Fig. 15. Thus, even for perfect alignment of the populations with each other (and a single fixed line of sight), the fractional errors on the estimated masses for the two populations may be significantly different, thus perturbing the inferred value of $\Gamma$, as a result of e.g. differences in the 3D shapes, levels of dispersion support or velocity dispersion anisotropies of the two populations. Considering the distribution of the standard deviation values, computed directly from the pixels shown in Fig. 15, the median value of the standard deviation (evenly weighted over the spherical surface) is

\footnotetext{
12 Ignoring from now on the claimed dependence on the stellar population concentration within the halo $\left(r_{\text {half }} / r_{\mathrm{DM}}\right)$, since this acts to make the NFW exclusion limits more conservative.
}

$\sigma\left[\alpha_{\text {Walker }}(\theta, \phi)\right]=0.140_{-0.028}^{+0.051}$, where the quoted range corresponds to the 16 th and 84 th percentiles. Thus, under the assumption of exact alignment of the populations, this value of $\sigma\left[\alpha_{\text {Walker }}(\theta, \phi)\right]$ could be taken as the scatter in the estimated masses for the individual populations, for a single randomly chosen line of sight. Yet clearly the expected scatter increases as the populations move away from the state of perfect alignment assumed in this special case, and the mean biases for different sightline orientations relative to the individual populations become relevant (see Fig. 14).

To represent the various uncertainties involved in estimating the enclosed masses for the two stellar populations, we assume in the following that, for each population, the estimated mass has an associated $1 \sigma$ fractional error of $\sigma_{\text {sys }}=0.2$. If the important properties of the two stellar populations are assumed to be independent, then the choice of $\sigma_{\text {sys }}=0.2$ seems reasonable in general given our analysis of the uncertainties involved in estimating the dynamical masses of realistic simulated galaxies in Section 5. This representative scatter of 20 per cent is relatively high compared to the standard deviations of the $\alpha$ values for most line-of-sight orientations relative to the stellar principal axes for the galaxies in our sample, although much larger scatters are observed for many viewing angles. Within the context of obtaining conservative exclusion confidences for NFWlike inner density profile slopes, our choice of $\sigma_{\text {sys }}$ might in fact be considered somewhat low; in which case the results presented below constitute an underestimate of the impact of the mass estimator errors on the reliability of the results of Walker \& Peñarrubia (2011). However, we are ignorant of any underlying correlations between the properties of the dual populations in Sculptor or Fornax that may conspire to bias the estimated enclosed masses in a similar way for the two radii, thus reducing the error on the inferred mass slope.

In order to assess the impact of the systematic errors inherent in simple mass estimators of the form assumed in equation (15) on the results of Walker \& Peñarrubia (2011), we begin by creating a simple model of their analysis procedure. Approximating the published posterior probability distribution functions for $\log _{10}\left(r_{2}\right)$, $r_{1} / r_{2}, \log _{10}\left(\sigma_{1}^{2}\right)$ and $\log _{10}\left(\sigma_{2}^{2}\right)$ as Gaussian (using the tabulated medians and 16 th-84th percentile ranges), we generate $10^{6}$ values for $\Gamma$ by independently drawing random values consistent with the four distributions, and applying equation (15). This approach ignores the correlations between the free parameters of the system, which are accounted for in the MCMC chains. However, the resulting $P(\Gamma)$ distributions for the two dSphs, shown in the upper panel of Fig. 17, are very similar to those obtained by Walker \& Peñarrubia (2011, cf. their fig. 10). Indeed, our median values for the slopes of $\Gamma=2.54_{-0.37}^{+0.44}$ for Fornax and $\Gamma=2.96_{-0.39}^{+0.49}$ for Sculptor are in excellent agreement with their results.

The lower panel of Fig. 17 shows the result of including a representative $1 \sigma$ fractional systematic error of $\sigma_{\text {sys }}=0.2$ in the mass estimates for both populations. In detail, we repeat the process described above, but for each set of radii and dispersions, we multiply $M\left(<r_{1}\right)$ and $M\left(<r_{2}\right)$ (or equivalently, the squared dispersions) by random values, $E_{1}$ and $E_{2}$, respectively, drawn separately from a Gaussian distribution centred at one with standard deviation $\sigma_{\text {sys }}$, and then proceed to apply equation (15) as before. ${ }^{13}$ Including the systematic scatter spreads out the $P(\Gamma)$ distributions, with a negligible impact on the median values, giving $\Gamma=2.54_{-0.70}^{+0.77}$ for Fornax and $\Gamma=2.95_{-0.61}^{+0.71}$ for Sculptor. In terms of the significance as defined in equation $(16)$, the $P(\Gamma)$ distributions including the

\footnotetext{
${ }^{13}$ We impose a lower limit of 0.01 for each of $E_{1}$ and $E_{2}$.
} 


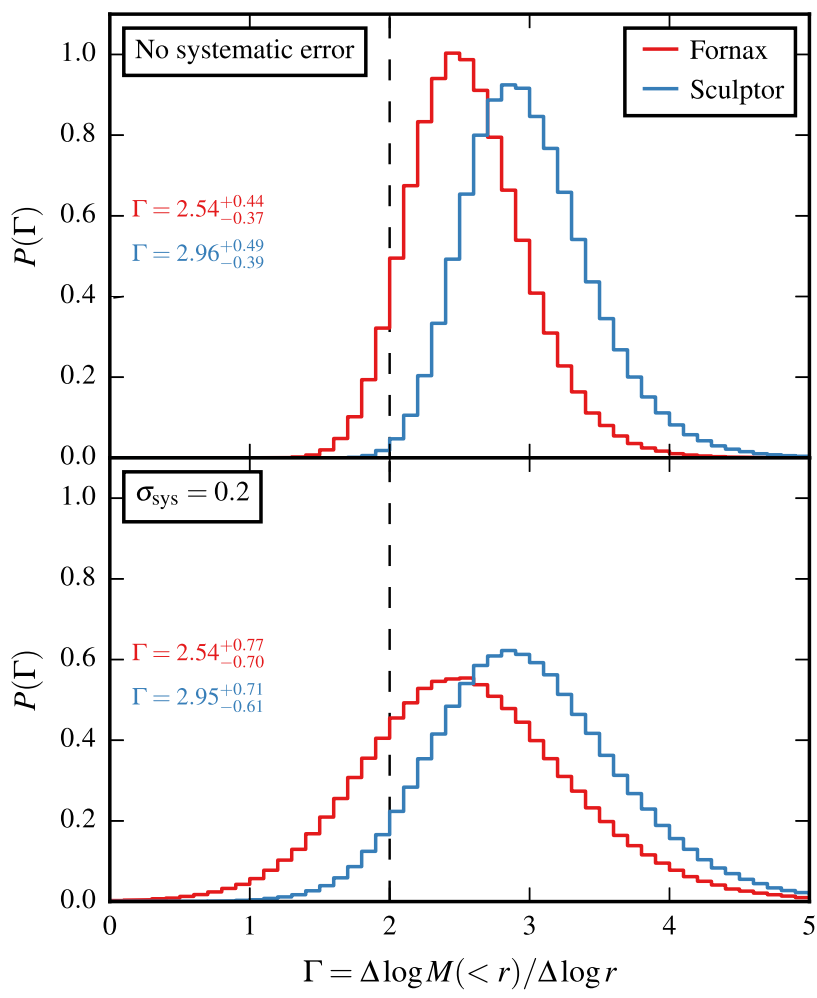

Figure 17. Distributions, $P(\Gamma)$, of the logarithmic mass profile slope, $\Gamma$, derived using chemo-dynamically distinct stellar subpopulations in Sculptor and Fornax (see equation 15). The upper panel shows the distributions from sampling the posterior probability distribution functions for the half-light radii and velocity dispersions from Walker \& Peñarrubia (2011). The lower panel shows the results of repeating this procedure, but introducing a $1 \sigma$ scatter of $\sigma_{\text {sys }}=0.2$ in the mass estimates for both populations. The median $\Gamma$ value and 16th-84th percentile confidence interval are given in each panel for each distribution, in the same colours as the lines. Each distribution is normalized such that $\int_{-\infty}^{\infty} P(\Gamma) \mathrm{d} \Gamma=1$. The vertical dashed line shows the central (maximum) mass slope for an NFW profile.

systematic error disfavour NFW slopes with confidence of only 77.7 and 94.5 per cent for Fornax and Sculptor, respectively. Clearly, the systematic errors associated with mass estimators of the form given in equation (1) have the potential to reduce the confidence with which it can be claimed that the results of Walker \& Peñarrubia (2011) rule out the presence of dark matter cusps as steep as that of the NFW profile in Fornax and Sculptor, depending on the extent to which the individual stellar populations are independent from each other.

A deeper understanding of the uncertainties associated with the methodology of Walker \& Peñarrubia (2011) will come from highresolution simulations where the chemo-dynamically distinct stellar populations in $\mathrm{dSphs}$ are treated in a realistic way, ideally as the result of cosmological initial conditions, such as in the APOSTLE simulation suite. Dwarf galaxies with multiple stellar populations may reside in our simulations, facilitating direct tests of methods that use estimators of enclosed dynamical masses to infer density slopes. Such analysis is beyond the scope of this paper; we leave the identification and classification of such galaxies, and analysis of their physical origins, to future studies using APOSTLE and similar high-resolution cosmological hydrodynamical simulations.

\section{AN OPTIMAL ESTIMATOR}

The mass estimators discussed in the preceding sections have a common form, as parametrized in equation (1), where $\lambda$ is the 3D radius within which the mass is estimated, in units of $R_{\mathrm{e}}$, and $\mu$ is the estimator prefactor. Extending this general idea, it may be that the radius within which the velocity dispersion is measured influences the accuracy of the recovered mass. Including an additional dimensionless parameter, $v$, we can write a more flexible generalization,

$M\left(<\lambda R_{\mathrm{e}}\right)=\frac{\mu\left\langle\sigma_{\mathrm{los}}\left(<v R_{\mathrm{e}}\right)\right\rangle^{2} R_{\mathrm{e}}}{G}$,

where $\left\langle\sigma_{\text {los }}(<R)\right\rangle$ is the line-of-sight stellar velocity dispersion measured within a projected radius, $R$, of the galactic centre. Up to now, we have averaged the velocity dispersion over the whole galaxy ( $v$ $\rightarrow \infty$ ), in keeping with the estimators proposed by Walker et al. (2009) and Wolf et al. (2010).

Setting aside the theoretical motivation for certain choices of the parameters in equation (17) which, at least for the estimators of Walker et al. (2009) and Wolf et al. (2010), are based on the assumptions that underpin the spherical Jeans equation combined with additional simplifications, we now ask: empirically, for the population of dispersion-dominated galaxies in our simulations, which set of estimator parameters yields an unbiased estimate of the true dynamical mass within some radius, with minimum scatter?

Considering our dispersion-dominated galaxy sample, the upper panel of Fig. 18 shows the standard deviation of the estimated to true mass ratio, $\alpha$, for the unbiased estimator obtained by setting $\mu$ such that the mean $\alpha$ is one for a given $\lambda$ and $\nu$. This grid has been computed by projecting over 1536 evenly distributed lines of sight, with a grid spacing of 0.01 in $\lambda$ and $\nu$. The scatter in the estimates varies strongly with $\lambda$, and depends more weakly on $v$. However, the impact of $v$ on the scatter becomes more significant near the location of minimum scatter in this plane (diamond marker). The middle panel in Fig. 18 shows the scatter as a function of $\lambda$ for the case where $v$ is chosen to minimize the scatter for each value of $\lambda$ (solid line), and also where the velocity dispersion averaged over the whole galaxy is used instead (dashed line). In the case where $v$ is allowed to vary, the scatter is minimized for $v \approx 1$, while including the whole galaxy in the dispersion measurement leads to a preference for higher $\lambda$.

The sets of parameters that minimize the scatter for these two treatments of $v$ are given in Table 3 , along with the associated scatter in the $\alpha$ distributions. We shall refer to the parameters found in the case where $v$ is allowed to vary as the 'optimum' set. The parameters in the case where the dispersion is measured within exactly $R_{\mathrm{e}}$ is also given in Table $3(v=1)$ and yield an equivalent level of scatter as in the optimum case. The uncertainties on the parameters and the scatter values given in Table 3 are derived from bootstrap resampling of the galaxies, where for each constraint on $v$, we draw $10^{4}$ samples of the same number of galaxies as in the true sample, and compute the 16th and 84th percentiles of the distributions of the parameters and scatter in $\alpha$ resulting from applying the minimization procedure to each sample. Fig. 19 shows the full $\alpha$ distribution in the optimum case. The reduction in the minimum scatter due to switching from $v \rightarrow \infty$ to the optimum parameter set is 7.0 per cent.

The mean $\alpha$ values and associated scatter obtained from applying the estimators of Walker et al. (2009) and Wolf et al. (2010) to the same galaxy sample as used in the calibration are shown in the lower panels of Fig. 18. Both of these estimators have a slightly 


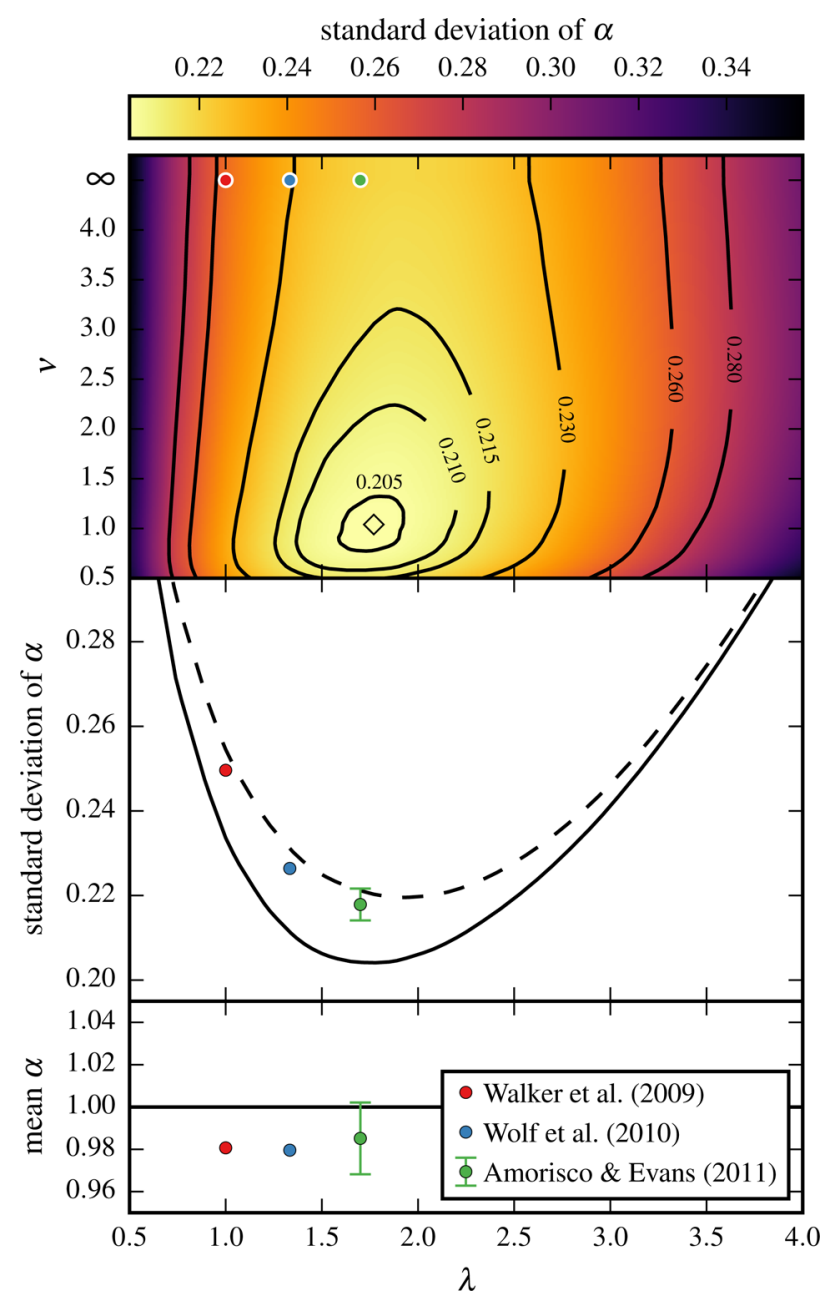

Figure 18. Calibration of mass estimator parameters $(\lambda, \mu, v)$ using all dispersion-dominated galaxies in our simulated sample (see equation 17). The upper panel shows the standard deviation of the estimated to true mass ratio $\alpha$ as a function of $\lambda$ and $\nu$, where the proportionality constant $\mu$ is chosen such that the estimator is unbiased (mean $\alpha$ equals one) for each combination of $\lambda$ and $\nu$. The scatter shown is linearly interpolated between $v=4$ and the case where the dispersion is averaged over the whole galaxy $(v \rightarrow \infty)$. The lines show contours of constant standard deviation, as labelled. The diamond symbol indicates the location of minimum scatter (see Table 3). The middle panel shows the scatter as a function of $\lambda$, for the choice of $v$ which minimizes the scatter at each value of $\lambda$ (solid line), and using instead the velocity dispersion averaged over the whole galaxy (dashed line). The points show the corresponding results from applying the estimators of Walker et al. (2009), Wolf et al. (2010) and Amorisco \& Evans (2011), which each use the dispersion averaged over the whole galaxy. Note that the $\alpha$ distribution for a given estimator can have a lower scatter than the dashed line, provided that it is biased in the mean. The lower panel shows the mean $\alpha$ for each of these three estimators.

smaller scatter than in the unbiased case that uses the dispersion averaged over the whole galaxy (as these two estimators do), for their $\lambda$. Using the optimum parameter set leads to reductions in the scatter of 18.2 and 9.9 per cent relative to the Walker et al. (2009) and Wolf et al. (2010) versions, respectively, while correcting for the bias exhibited by each estimator, as shown in Fig. 18.

We note that Amorisco \& Evans (2011) obtain an empirical estimator with similar parameters to our optimum result, from phasespace modelling of the dSphs of the MW (they find $\lambda=1.7$ and
Table 3. Parameters $(\lambda, \mu, v)$ that yield an estimated to true mass ratio, $\alpha$, of one on average, with minimum scatter, calibrated using all galaxies in our dispersion-dominated sample (see equation 17). The three sets of parameters shown result from different constraints on $v$, which sets the radius within which the velocity dispersion is measured. The final row gives the scatter for each parameter set (standard deviation of $\alpha$ ). The quoted uncertainties are the 16th-84th percentile confidence limits, from $10^{4}$ bootstrap samples of the galaxies, for each constraint on $v$.

\begin{tabular}{lccc}
\hline Parameter & $\begin{array}{c}0.1<v<4.0 \\
\text { (optimum set) }\end{array}$ & $v=1$ & $v \rightarrow \infty$ \\
\hline$\lambda$ & $1.77_{-0.06}^{+0.08}$ & $1.76_{-0.05}^{+0.09}$ & $1.91_{-0.03}^{+0.09}$ \\
$\mu$ & $5.99_{-0.33}^{+0.37}$ & $5.94_{-0.28}^{+0.42}$ & $6.95_{-0.18}^{+0.48}$ \\
$\nu$ & $1.04_{-0.17}^{+0.10}$ & 1 & $\infty$ \\
\hline Scatter & $0.204_{-0.006}^{+0.005}$ & $0.204_{-0.006}^{+0.005}$ & $0.220_{-0.006}^{+0.005}$ \\
\hline
\end{tabular}

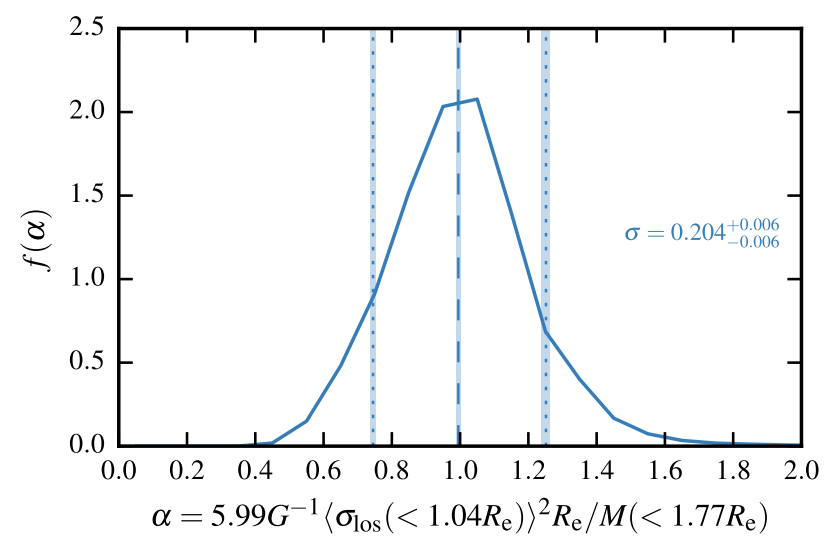

Figure 19. Distribution, $f(\alpha)$, of the estimated to true mass ratio, $\alpha$, for the unbiased estimator with minimum scatter of the form given in equation (17). The optimum parameters are given in Table 3 (as in the horizontal axis label). The distribution considers all dispersion-dominated galaxies $\left(\kappa_{\text {rot }}<0.5\right)$, using projections from 1536 evenly distributed lines of sight. The dashed line shows the median, and the dotted lines show the 10th and 90th percentiles. The standard deviation, $\sigma$, is also shown. The shaded regions around the vertical lines and the quoted errors on $\sigma$ are the 16th-84th percentile confidence limits, derived from applying the estimator to $10^{4}$ bootstrap samples of the galaxies. Note that the similar $\sigma$ errors given in Table 3 for this estimator are instead derived from the distribution of optimum $\sigma$ values obtained from repeatedly calibrating equation (17) on bootstrap samples of the same galaxies. Each projection of each galaxy contributes to the distribution with equal weight, and the distribution is normalized to have unit area.

$\mu=5.8 \pm 1.0$; cf. Table 3 ). The choice of $1.7 R_{\mathrm{e}}$ for the enclosing radius minimizes the dependence in their analysis of the recovered mass on the assumed halo density profile and its associated scalelength, assuming that the $\mathrm{dSphs}$ have isotropic velocity dispersions in their centres, with relatively flat $\sigma_{\text {los }}(R)$ profiles. The mean $\alpha$ and scatter resulting from applying this estimator to our dispersiondominated sample are shown in Fig. 18 alongside the Walker et al. (2009) and Wolf et al. (2010) results, where the error bars indicate the spread due to the quoted uncertainty on $\mu .^{14}$

\footnotetext{
${ }^{14}$ Amorisco \& Evans (2011) state that their analysis uses the velocity dispersion at the galactic centre; however, it is not clear to what level the dispersions used can be considered to be 'central'. The data set used is that
} 


\section{CONCLUSIONS}

In this paper, we have presented the key intrinsic and observable properties of galaxies drawn from the APOSTLE simulations of the Local Group that are relevant to Jeans analysis in general, and in particular to the use of simple mass estimators of the form given in equation (1), as advocated by Walker et al. (2009) and Wolf et al. (2010). The simulated galaxies have realistic stellar density distributions and line-of-sight stellar velocity dispersions, combined with a range of 3D shapes, levels of dispersion support and stellar velocity dispersion anisotropy.

Applying the mass estimators proposed by Walker et al. (2009) and Wolf et al. (2010) to many projections of each galaxy in our sample, we have found that each estimator has a small bias in the estimated to true mass ratio, $\alpha$, combined with a relatively large scatter. This scatter is 23 or 25 per cent at the $1 \sigma$ level overall for dispersion-dominated galaxies, respectively (see Fig. 9).

The dependence of $\alpha$ on various galaxy properties is summarized in Fig. 16. The scatter in $\alpha$ depends strongly on the shape of the galaxy, such that the $\alpha$ distributions are sharply peaked for stellar mass distributions that are close to spherical symmetry. However, the dependence of the scatter on the observable 2D shape on the sky is much weaker, i.e. it is difficult to identify ideal spherically symmetric systems in projection. The scatter also increases for progressively larger radial bias in the stellar velocity dispersion anisotropy, progressively higher levels of rotational support, and for galaxies that exhibit closer alignment with their host dark matter haloes. We also find that the scatter is lowest for galaxies that have relatively low stellar kinetic energy for their gravitational potential energy, and for galaxies that are highly extended, in units of their halo scale radius. The mass estimator accuracy is more sensitive to the $3 \mathrm{D}$ shape (and alignment with respect to the observer) of the galaxy than to that of the host dark matter halo. The dispersion-dominated satellites of the MW and M31 analogues in the simulations yield a smaller scatter in $\alpha$ (typically 20-22 per cent) than the dispersiondominated field galaxies (typically $24-26$ per cent), as shown in Fig. 12.

Considering the dependence of $\alpha$ on the orientation of the line of sight with respect to the galaxy, we find that the dynamical masses are overestimated with a relatively large scatter for lines of sight coincident with the stellar morphological major axis, underestimated with a modest scatter along the minor axis, and close to accurate with a modest scatter for the observations along the stellar principal axis of intermediate length (see Figs 14 and 15).

Adopting a representative value of 20 per cent for the $1 \sigma$ systematic scatter in $\alpha$ for satellite galaxies, we have shown that the systematic uncertainties inherent in the simple estimators significantly increase the errors on the estimated dynamical masses of dSph satellites of the MW from Wolf et al. (2010) in cases where the observational errors on the half-light radii and velocity dispersions are relatively small. Including the same representative scatter within a simple model of the analysis of Walker \& Peñarrubia (2011) demonstrates that systematic uncertainties can also greatly reduce the significance with which the mass profile slopes derived for two independent stellar populations in Sculptor and Fornax are inconsistent with an NFW dark matter density profile. This result depends on the level to which the errors on the

compiled by Walker et al. (2009, 2010), who note that the dispersions are global averages. Therefore, we use the velocity dispersion averaged over the whole galaxy when applying the estimator of Amorisco \& Evans (2011), in order to be consistent with the data used in their analysis. estimated masses for the two populations can be assumed to be independent.

Finally, we have investigated the scatter in the estimated to true mass ratio for the set of unbiased estimators of the form given in equation (17), considering all the dispersion-dominated galaxies in our simulated sample. We find that using the optimum set of parameters given in Table 3 results in a reduction in the scatter of between 10 and 20 per cent with respect to similar estimators in the literature (Walker et al. 2009; Wolf et al. 2010; Amorisco \& Evans 2011), combined with the removal of bias in the mean. The scatter is optimized when the line-of-sight velocity dispersion is measured within a radius close to the projected stellar half-mass radius, $R_{\mathrm{e}}$ (rather than over the whole galaxy as is typical):

$M\left(<1.77 R_{\mathrm{e}}\right)=\frac{5.99\left\langle\sigma_{\mathrm{los}}\left(<1.04 R_{\mathrm{e}}\right)\right\rangle^{2} R_{\mathrm{e}}}{G}$.

This equation gives our optimum result in the case where the radius within which the velocity dispersion is measured is treated as a free parameter. If instead we fix the dispersion measurement to consider stars within exactly $R_{\mathrm{e}}$, the resulting scatter is the same as that obtained using the formally optimal parameter set (see Table 3).

\section{ACKNOWLEDGEMENTS}

We would like to thank Mark Wilkinson and Manoj Kaplinghat for interesting discussions, along with Louie Strigari, who provided the MW dSph profiles. We would also like to thank the referee, Matt Walker, for helpful comments and suggestions that have improved the paper. This work was supported by the Science and Technology Facilities Council (grant number ST/L00075X/1) and the European Research Council (grant number GA 267291, 'Cosmiway'). DJRC acknowledges the support of STFC studentship ST/K501979/1. This work used the DiRAC Data Centric system at Durham University, operated by the Institute for Computational Cosmology on behalf of the STFC DiRAC HPC Facility (www.dirac.ac.uk). This equipment was funded by BIS National E-infrastructure capital grant ST/K00042X/1, STFC capital grants ST/H008519/1 and ST/K00087X/1, STFC DiRAC Operations grant ST/K003267/1 and Durham University. DiRAC is part of the National E-Infrastructure.

\section{REFERENCES}

Amorisco N. C., Evans N. W., 2011, MNRAS, 411, 2118

Barber C., Starkenburg E., Navarro J. F., McConnachie A. W., 2015, MNRAS, 447, 1112

Battaglia G., Helmi A., Tolstoy E., Irwin M., Hill V., Jablonka P., 2008, ApJ, 681, L13

Benítez-Llambay A., Navarro J. F., Abadi M. G., Gottlöber S., Yepes G., Hoffman Y., Steinmetz M., 2016, MNRAS, 456, 1185

Bett P., 2012, MNRAS, 420, 3303

Binney J., Mamon G. A., 1982, MNRAS, 200, 361

Booth C. M., Schaye J., 2009, MNRAS, 398, 53

Boylan-Kolchin M., Bullock J. S., Kaplinghat M., 2011, MNRAS, 415, L40

Coleman M. G., Da Costa G. S., Bland-Hawthorn J., Freeman K. C., 2005, AJ, 129, 1443

Crain R. A. et al., 2015, MNRAS, 450, 1937

Dalla Vecchia C., Schaye J., 2012, MNRAS, 426, 140

Davis M., Efstathiou G., Frenk C. S., White S. D. M., 1985, ApJ, 292, 371

Diemand J., Kuhlen M., Madau P., Zemp M., Moore B., Potter D., Stadel J., 2008, Nature, 454, 735 
Dolag K., Borgani S., Murante G., Springel V., 2009, MNRAS, 399, 497

Fattahi A. et al., 2016, MNRAS, 457, 844

Górski K. M., Hivon E., Banday A. J., Wandelt B. D., Hansen F. K., Reinecke M., Bartelmann M., 2005, ApJ, 622, 759

Grand R. J. J., Springel V., Gómez F. A., Marinacci F., Pakmor R., Campbell D. J. R., Jenkins A., 2016, MNRAS, 459, 199

Hopkins P. F., 2013, MNRAS, 428, 2840

Irwin M., Hatzidimitriou D., 1995, MNRAS, 277, 1354

Komatsu E. et al., 2011, ApJS, 192, 18

Kowalczyk K., Łokas E. L., Kazantzidis S., Mayer L., 2013, MNRAS, 431, 2796

Laporte C. F. P., Walker M. G., Peñarrubia J., 2013a, MNRAS, 433, L54

Laporte C. F. P., White S. D. M., Naab T., Gao L., 2013b, MNRAS, 435, 901

Lyskova N., Thomas J., Churazov E., Tremaine S., Naab T., 2015, MNRAS, 450,3442

McConnachie A. W., 2012, AJ, 144, 4

Muñoz R. R. et al., 2006, ApJ, 649, 201

Navarro J. F., Frenk C. S., White S. D. M., 1996, ApJ, 462, 563

Odenkirchen M. et al., 2001, AJ, 122, 2538

Peñarrubia J., McConnachie A. W., Navarro J. F., 2008a, ApJ, 672, 904

Peñarrubia J., McConnachie A. W., Navarro J. F., 2008b, ApJ, 687, 1460

Rosas-Guevara Y. M. et al., 2015, MNRAS, 454, 1038

Sales L. V., Navarro J. F., Theuns T., Schaye J., White S. D. M., Frenk C. S., Crain R. A., Dalla Vecchia C., 2012, MNRAS, 423, 1544

Sawala T. et al., 2016, MNRAS, 457, 1931

Schaller M., Dalla Vecchia C., Schaye J., Bower R. G., Theuns T., Crain R. A., Furlong M., McCarthy I. G., 2015, MNRAS, 454, 2277

Schaye J., 2004, ApJ, 609, 667

Schaye J., Dalla Vecchia C., 2008, MNRAS, 383, 1210

Schaye J. et al., 2015, MNRAS, 446, 521

Smolčić V., Zucker D. B., Bell E. F., Coleman M. G., Rix H. W., Schinnerer E., Ivezić Ž., Kniazev A., 2007, AJ, 134, 1901

Springel V., 2005, MNRAS, 364, 1105

Springel V., White S. D. M., Tormen G., Kauffmann G., 2001, MNRAS, 328,726

Springel V. et al., 2008, MNRAS, 391, 1685

Strigari L. E., Bullock J. S., Kaplinghat M., Diemand J., Kuhlen M., Madau P., 2007, ApJ, 669, 676

Strigari L. E., Bullock J. S., Kaplinghat M., Simon J. D., Geha M., Willman B., Walker M. G., 2008, Nature, 454, 1096

Strigari L. E., Frenk C. S., White S. D. M., 2010, MNRAS, 408, 2364

Strigari L. E., Frenk C. S., White S. D. M., 2014, AAS, preprint (arXiv:1406.6079)

Subramanian S., Subramaniam A., 2012, ApJ, 744, 128

Walker M. G., Peñarrubia J., 2011, ApJ, 742, 20

Walker M. G., Mateo M., Olszewski E. W., Peñarrubia J., Wyn Evans N., Gilmore G., 2009, ApJ, 704, 1274

Walker M. G., Mateo M., Olszewski E. W., Peñarrubia J., Wyn Evans N., Gilmore G., 2010, ApJ, 710, 886

Weinberg M. D., Nikolaev S., 2001, ApJ, 548, 712

Wetzel A. R., Hopkins P. F., Kim J.-h., Faucher-Giguère C.-A., Kereš D., Quataert E., 2016, ApJ, 827, L23

Wiersma R. P. C., Schaye J., Smith B. D., 2009a, MNRAS, 393, 99

Wiersma R. P. C., Schaye J., Theuns T., Dalla Vecchia C., Tornatore L., 2009b, MNRAS, 399, 574

Wolf J., Martinez G. D., Bullock J. S., Kaplinghat M., Geha M., Muñoz R. R., Simon J. D., Avedo F. F., 2010, MNRAS, 406, 1220

\section{APPENDIX A: WOLF ET AL.(2010) ESTIMATOR IN 3D}

In the main body of this paper, we have made use of the estimator of Wolf et al. (2010) expressed in terms of observable quantities, i.e. the projected stellar half-light (half-mass) radius, $R_{\mathrm{e}}$, and lineof-sight velocity dispersion, $\left\langle\sigma_{\text {los }}\right\rangle$ (equation 9). However, a more fundamental version of the estimator is given in equation (8), from which Wolf et al. (2010) derive equation (9) by simply assuming that the $3 \mathrm{D}$ half-light radius, $r_{\text {half }}$, is $4 R_{\mathrm{e}} / 3$, which is a reasonable approximation for a range of spherically symmetric density profiles. Yet the appropriateness of this assumption could vary significantly for some galaxies, depending on the shapes of their stellar density profiles. We now investigate the change in the accuracy of the estimator of Wolf et al. (2010), with respect to the standard case given in equation (9), when (i) a value for $r_{\text {half }}$ is inferred from the projected stellar density distribution, instead of assuming $r_{\text {half }}=4 R_{\mathrm{e}} / 3$, and (ii) typically unobservable $3 \mathrm{D}$ information on the galaxy size and velocity dispersion is used in the mass estimation.

\section{A1 Deprojection of the stellar mass profile}

In order to assess whether the assumption, $r_{\text {half }}=4 R_{\mathrm{e}} / 3$, is optimal for the dispersion-dominated galaxies in our simulated sample, which have a broad range of 3D shapes, we carry out an Abel inversion of the projected mass distribution for all 1536 projections of each galaxy. We use splines to model the cumulative projected stellar mass profile, $M_{\text {star }}(<R)$. This choice allows the model profile to be quite general in form, in the sense that the inference of $r_{\text {half }}$ should not suffer from any overly restrictive parametrization that may bias the results. In detail, we find the quartic ${ }^{15}$ spline that interpolates exactly through points of even spacing, $\Delta R$, on the $M_{\text {star }}(<R)$ profile, starting from the galactic centre. The profile is reflected about the centre before fitting, so that the model is well behaved in the innermost regions. Additionally, we extend $M_{\text {star }}(<R)$ for several $\Delta R$ beyond the furthest star particle (where it equals the total stellar mass, $M_{\text {star }}$ ). Starting with an initial spacing of $\Delta R=R_{\mathrm{e}} / 2$, we recursively multiply $\Delta R$ by 0.95 and refit the spline until the following condition is satisfied,

$\frac{1}{\sigma_{\text {fit }}^{2}} \sum_{i}\left[M_{\text {model }}\left(<R_{i}\right)-M_{\text {star }}\left(<R_{i}\right)\right]^{2}<n_{\text {star }}$,

where the sum is over all star particles in the galaxy, with projected radii $R_{i} . M_{\text {model }}(<R)$ is the stellar mass enclosed within $R$ according to the spline fit, and $n_{\text {star }}$ is the number of star particles. The choice of the parameter $\sigma_{\text {fit }}=0.01 M_{\text {star }}$ represents a compromise between the smoothness and accuracy of the fit.

Given a model for $M_{\text {star }}(<R)$, and assuming spherical symmetry, the 3D stellar density profile, $\rho(r)$, follows from the Abel integral given in equation (5), where,

$$
\frac{\mathrm{d} \Sigma(R)}{\mathrm{d} R}=\frac{1}{2 \pi R}\left[\frac{\mathrm{d}^{2} M_{\text {star }}(<R)}{\mathrm{d} R^{2}}-\frac{1}{R} \frac{\mathrm{d} M_{\text {star }}(<R)}{\mathrm{d} R}\right] .
$$

The equations can then be solved numerically for $r_{\text {half }}$.

In Fig. A1, we show the distribution of the ratio of the estimated 3D half-mass radius from our deprojection procedure, $r_{\text {deproj }}$, to the true radius, $r_{\text {half }}$, for all galaxies in our dispersion-dominated sample, alongside the corresponding distribution from assuming that $r_{\text {half }}$ is simply $4 R_{\mathrm{e}} / 3$. The distributions are remarkably similar, and show that overall there is no improvement in the approximation of the 3D size when switching from using the factor of four thirds

\footnotetext{
${ }^{15}$ So that the second derivative is both continuous and smooth.
} 


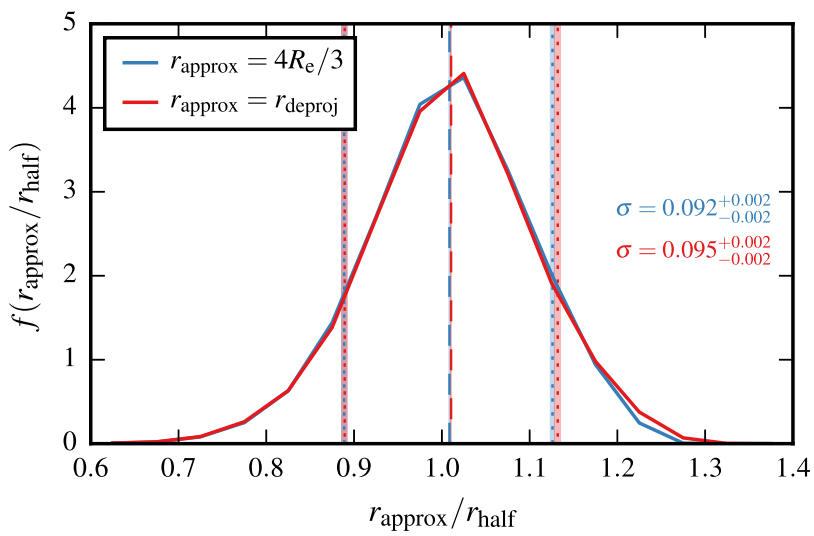

Figure A1. Distributions, $f\left(r_{\text {approx }} / r_{\text {half }}\right)$, of the ratio of approximations, $r_{\text {approx }}$, for the 3D half-mass radius, $r_{\text {half }}$, to the true value of the radius for galaxies in our dispersion-dominated sample $\left(\kappa_{\text {rot }}<0.5\right)$. The $r_{\text {approx }}$ values are computed for 1536 evenly distributed lines of sight, assuming either that $r_{\text {half }}=4 R_{\mathrm{e}} / 3$, or using $r_{\text {deproj }}$ from our Abel deprojection procedure (different colours). Each projection of each galaxy contributes to the distributions with equal weight, and each distribution is normalized to have unit area. The vertical dashed lines show the median ratios, and the dotted lines show the 10th and 90th percentiles. The standard deviation, $\sigma$, of each distribution is given in the same colour as the lines. The shaded regions around the vertical lines and the $\sigma$ errors are the 16th-84th percentile confidence limits, derived from $10^{4}$ bootstrap samples of the galaxies for each distribution.

to using our deprojection procedure (the scatter actually increases slightly for $r_{\text {deproj }}$ ).

In Fig. A2(a) we show the distribution of the estimated to true mass ratio $\alpha$ for all dispersion-dominated galaxies in our simulated sample, obtained using the standard projected version of the Wolf et al. (2010) estimator from equation (9) (as in the upper panel of Fig. 9). Fig. A2(b) shows the distribution if instead we use the 3D version of the estimator from equation (8), replacing $r_{\text {half }} \rightarrow r_{\text {deproj }}$. There is no significant difference between the distributions shown in panels (a) and (b), and so we conclude that assuming $r_{\text {half }}=4 R_{\mathrm{e}} / 3$ is already optimal, despite the scatter shown in Fig. A1.

\section{A2 Using true 3D galaxy properties}

It is interesting to ask how the accuracy of the estimator of Wolf et al. (2010) changes if intrinsic 3D, i.e. typically observationally inaccessible, information is available on the galaxy sizes and kinematics. Fig. A2(c) shows the distribution of the estimated to true mass ratio when using the true $3 \mathrm{D}$ half-mass radius in equation (8), so the only projected quantity used to compute the distribution shown is $\left\langle\sigma_{\text {los }}\right\rangle$. The removal of the noise due to the variation of the projected size reduces the scatter in the distributions, comparing to panels (a) and (b).

In Fig. A2(d), we show the distribution of the ratio of the estimated mass from equation (9), i.e. the version of estimator that uses $R_{\mathrm{e}}$, to the true mass within $r_{\text {half }}$. Thus, here $\alpha$ is not the ratio of the estimated mass to the true mass within the same sphere, as everywhere else in this paper. The scatter in this distribution is lower than in panel (c), where the only difference in the definition of $\alpha$ is the replacement $3 r_{\text {half }} \rightarrow 4 R_{\mathrm{e}}$ from (c) to (d). That is, equation (9) is a better estimator of the mass within the true $3 \mathrm{D}$ half-mass radius than equation (8). This result may seem counterintuitive at first, as the former makes use of only projected information to estimate the mass, while the true 3D half-mass radius is used in the latter case. This effect can be understood in terms of the coupled variation of $R_{\mathrm{e}}$ and $\left\langle\sigma_{\text {los }}\right\rangle$ over lines of sight which view the galaxy from many orientations, such that the variation in the projected size counteracts that in the (squared) dispersion, so that the product $4\left\langle\sigma_{\text {los }}\right\rangle^{2} R_{\mathrm{e}}$ has a smaller scatter than $3\left\langle\sigma_{\text {los }}\right\rangle^{2} r_{\text {half }}$ for the vast majority of galaxies (see Fig. 13 and also Laporte et al. 2013a). The result that

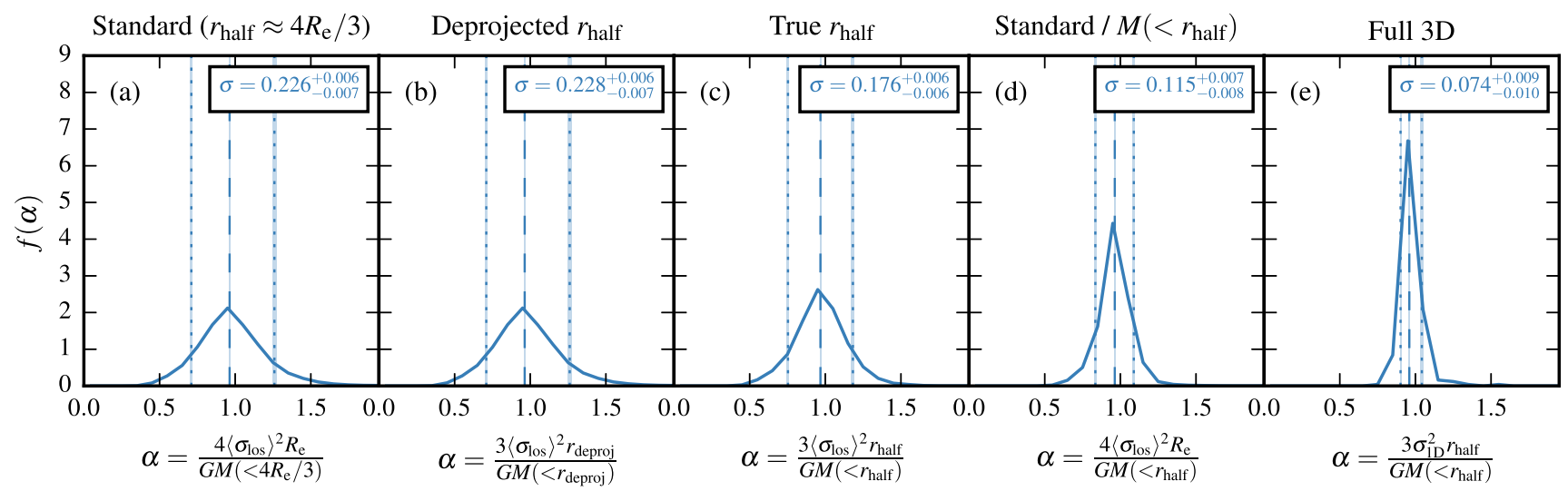

Figure A2. Distributions, $f(\alpha)$, of the estimated to true mass ratio, $\alpha$, for all dispersion-dominated galaxies $\left(\kappa_{\text {rot }}<0.5\right)$, obtained from projecting over 1536 evenly distributed lines of sight, using different versions of the estimator proposed by Wolf et al. (2010). The definition of $\alpha$ is different in each panel, and is given explicitly in each horizontal axis label. The definitions are (a) the ratio of the estimated mass using equation (9) to the mass enclosed within the assumed 3D half-mass radius $\left(4 R_{\mathrm{e}} / 3\right)$ - this is the standard definition of $\alpha_{\text {Wolf }}$ as given in equation (14), (b) the estimated to true mass ratio from equation (8) using the deprojected 3D half-mass radius, $r_{\mathrm{deproj}}$, from our Abel integration procedure in place of $r_{\text {half }}$, (c) the same as (b) but using the true 3D half-mass radius, $r_{\text {half }}$, (d) the ratio of the estimated mass from equation (9) to the mass within $r_{\text {half }}$ (i.e. this is the ratio of the estimated to true mass within different spheres, unless $r_{\text {half }}=4 R_{\mathrm{e}} / 3$ ) and (e) the estimated to true mass ratio if we replace $\left\langle\sigma_{\operatorname{los}}\right\rangle$ in equation (8) with the mean $1 \mathrm{D}$ stellar velocity dispersion, $\sigma_{1 \mathrm{D}}=\sigma_{3 \mathrm{D}} / \sqrt{3}$ (so no projected information is used in this panel). Each projection (if applicable) of each galaxy contributes to the distributions with equal weight, and each distribution is normalized to have unit area. The vertical dashed lines show the median $\alpha$ values, and the dotted lines show the 10th and 90th percentiles. The standard deviation, $\sigma$, of each distribution is given in each panel. The shaded regions around the vertical lines and the $\sigma$ errors are the 16th-84th percentile confidence limits, derived from $10^{4}$ bootstrap samples of the galaxies for each distribution. 
equation (9) is a better estimator of $M\left(<r_{\text {half }}\right)$ than equation (8) is only attractive if for some reason one wanted to know $M\left(<r_{\text {half }}\right)$ without any associated estimate of $r_{\text {half }}$ itself. When full 3D information is available on the stellar velocities, so that we can replace $\left\langle\sigma_{\text {los }}\right\rangle$ in equation (8) with the mean $1 \mathrm{D}$ dispersion, $\sigma_{1 \mathrm{D}}=\sigma_{3 \mathrm{D}} / \sqrt{3}$, the scatter in the estimated masses reduces significantly, as shown in Fig. A2(e). Note that no projected information is used in this panel, so each galaxy contributes a single value to the distribution.

This paper has been typeset from a $\mathrm{T}_{\mathrm{E}} \mathrm{X} / \mathrm{L} \mathrm{T}_{\mathrm{E}} \mathrm{X}$ file prepared by the author. 